\title{
Analysis of the $\pi^{+} \pi^{-} \pi^{+} \pi^{-}$and $\pi^{+} \pi^{0} \pi^{-} \pi^{0}$ final states in quasi-real two-photon collisions at LEP
}

\section{L3 Collaboration}

P. Achard ${ }^{\mathrm{t}}$, O. Adriani ${ }^{\mathrm{q}}$, M. Aguilar-Benitez ${ }^{\mathrm{y}}, \mathrm{J}$. Alcaraz ${ }^{\mathrm{y}}$, G. Alemanni ${ }^{\mathrm{w}}$, J. Allaby ${ }^{\mathrm{r}}$, A. Aloisio ${ }^{\text {ac }}$, M.G. Alviggi ${ }^{\text {ac }}$, H. Anderhub ${ }^{\text {aw }}$, V.P. Andreev ${ }^{\text {f,ah }}$, F. Anselmo ${ }^{\text {h }}$, A. Arefiev ${ }^{\text {ab }}$, T. Azemoon ${ }^{c}$, T. Aziz ${ }^{i}$, P. Bagnaia ${ }^{\text {am }}$, A. Bajo ${ }^{y}$, G. Baksay ${ }^{z}$, L. Baksay ${ }^{z}$, S.V. Baldew ${ }^{\text {b }}$, S. Banerjee ${ }^{\text {i }}$, Sw. Banerjee ${ }^{\text {d}}$, A. Barczyk ${ }^{\text {aw,au }}$, R. Barillère ${ }^{\mathrm{r}}$, P. Bartalini ${ }^{\mathrm{w}}$, M. Basile ${ }^{\mathrm{h}}$, N. Batalova ${ }^{\text {at }}$, R. Battiston ${ }^{\text {ag }}$, A. Bay ${ }^{\text {w }}$, F. Becattini ${ }^{\text {q, }}$ U. Becker ${ }^{\mathrm{m}}$, F. Behner ${ }^{\text {aw }}$, L. Bellucci ${ }^{\mathrm{q}}$, R. Berbeco ${ }^{\mathrm{c}}$, J. Berdugo ${ }^{y}$, P. Berges ${ }^{\mathrm{m}}$, B. Bertucci ${ }^{\mathrm{ag}}$, B.L. Betev ${ }^{\text {aw }}$, M. Biasini ${ }^{\mathrm{ag}}$, M. Biglietti ${ }^{\text {ac }}$, A. Biland ${ }^{\text {aw }}$, J.J. Blaising ${ }^{\text {d }, ~ S . C . ~ B l y t h ~}{ }^{\text {ai }}$, G.J. Bobbink ${ }^{\text {b }}$, A. Böhm ${ }^{\text {a }}$, L. Boldizsar ${ }^{1}$, B. Borgia ${ }^{\text {am }}$, S. Bottai ${ }^{\text {q, }}$ D. Bourilkov ${ }^{\text {aw }}$, M. Bourquin ${ }^{\mathrm{t}}$, S. Braccini ${ }^{\mathrm{t}}$, J.G. Branson ${ }^{\text {ao }}$, F. Brochu ${ }^{\mathrm{d}}$, J.D. Burger ${ }^{\mathrm{m}}$, W.J. Burger ${ }^{\text {ag }}$, X.D. Cai ${ }^{\mathrm{m}}$, M. Capell ${ }^{\mathrm{m}}$, G. Cara Romeo ${ }^{\mathrm{h}}$, G. Carlino ${ }^{\mathrm{ac}}$, A. Cartacci ${ }^{\mathrm{q}}$, J. Casaus ${ }^{\mathrm{y}}$, F. Cavallari ${ }^{\text {am }}$, N. Cavallo ${ }^{\text {aj }}$, C. Cecchi ${ }^{\text {ag }}$, M. Cerrada ${ }^{\mathrm{y}}$, M. Chamizo ${ }^{\mathrm{t}}$, Y.H. Chang ${ }^{\text {ar }}$, M. Chemarin ${ }^{\mathrm{x}}$, A. Chen ${ }^{\text {ar }}$, G. Chen ${ }^{\mathrm{g}}$, G.M. Chen ${ }^{\mathrm{g}}$, H.F. Chen ${ }^{\mathrm{v}}$, H.S. Chen ${ }^{\mathrm{g}}$, G. Chiefari ${ }^{\mathrm{ac}}$, L. Cifarelli ${ }^{\text {an }}$, F. Cindolo ${ }^{\text {h}}$, I. Clare ${ }^{\mathrm{m}}$, R. Clare ${ }^{\mathrm{al}}$, G. Coignet ${ }^{\mathrm{d}}$, N. Colino ${ }^{\mathrm{y}}$, S. Costantini $^{\mathrm{am}}$, B. de la Cruz ${ }^{\mathrm{y}}$, S. Cucciarelli ag, R. de Asmundis ${ }^{\text {ac }}$, P. Déglon ${ }^{t}$, J. Debreczeni ${ }^{1}$, A. Degré ${ }^{d}$, K. Dehmelt ${ }^{z}$, K. Deiters ${ }^{\text {au }}$, D. della Volpe ${ }^{\text {ac }}$, E. Delmeire ${ }^{\mathrm{t}}$, P. Denes ${ }^{\mathrm{ak}}$, F. De Notaristefani ${ }^{\mathrm{am}}$, A. De Salvo ${ }^{\text {aw }}$,

M. Diemoz ${ }^{a m}$, M. Dierckxsens ${ }^{b}$, C. Dionisi ${ }^{a m}$, M. Dittmar ${ }^{\text {aw }}$, A. Doria ${ }^{a c}$, M.T. Dova ${ }^{\mathrm{j}, 1}$, D. Duchesneau ${ }^{\text {d }}$, M. Duda ${ }^{\text {a }}$, B. Echenard ${ }^{\text {t }}$, A. Eline ${ }^{\mathrm{r}}$, A. El Hage ${ }^{\mathrm{a}}$, H. El Mamouni ${ }^{\mathrm{x}}$, A. Engler ${ }^{\text {ai }}$, F.J. Eppling ${ }^{\mathrm{m}}$, P. Extermann ${ }^{\mathrm{t}}$, M.A. Falagan ${ }^{\mathrm{y}}$, S. Falciano ${ }^{\text {am }}$, A. Favara ${ }^{\text {af }}$, J. Fay ${ }^{\mathrm{x}}$, O. Fedin ${ }^{\text {ah }}$, M. Felcini ${ }^{\text {aw }}$, T. Ferguson ${ }^{\text {ai }}$, H. Fesefeldt ${ }^{a}$, E. Fiandrini ${ }^{\text {ag }}$, J.H. Field ${ }^{t}$, F. Filthaut ${ }^{\text {ae }}$, P.H. Fisher ${ }^{\mathrm{m}}$, W. Fisher ${ }^{\text {ak }}$, G. Forconi ${ }^{\mathrm{m}}$, K. Freudenreich ${ }^{\text {aw }}$, C. Furetta ${ }^{\text {aa }}$, Yu. Galaktionov ${ }^{\text {ab,m }}$, S.N. Ganguli ${ }^{\mathrm{i}}$, P. Garcia-Abia ${ }^{y}$, M. Gataullin ${ }^{\text {af }}$, S. Gentile ${ }^{\text {am }}$, S. Giagu ${ }^{\text {am }}$, Z.F. Gong ${ }^{\text {v }}$, G. Grenier ${ }^{\mathrm{x}}$, O. Grimm ${ }^{\text {aw }}$, M.W. Gruenewald ${ }^{\mathrm{p}}$, M. Guida ${ }^{\text {an }}$, V.K. Gupta ${ }^{\text {ak }}$, A. Gurtu ${ }^{\mathrm{i}}$, L.J. Gutay ${ }^{\text {at }}$, D. Haas ${ }^{\mathrm{e}}$,

D. Hatzifotiadou ${ }^{\text {h }}$, T. Hebbeker ${ }^{\text {a }}$, A. Hervé ${ }^{\text {, J. Jirschfelder }}{ }^{\text {ai }}$, H. Hofer aw , M. Hohlmann ${ }^{\text {z }}$, G. Holzner ${ }^{\text {aw }}$, S.R. Hou ${ }^{\text {ar }}$, B.N. Jin ${ }^{\mathrm{g}}$, P. Jindal ${ }^{\mathrm{n}}$, L.W. Jones ${ }^{\mathrm{c}}$, P. de Jong ${ }^{\mathrm{b}}$, I. Josa-Mutuberría ${ }^{\mathrm{y}}$, M. Kaur ${ }^{\mathrm{n}}$, M.N. Kienzle-Focacci ${ }^{\mathrm{t}}$, J.K. Kim ${ }^{\text {aq }}$, J. Kirkby ${ }^{\mathrm{r}}$, W. Kittel ${ }^{\text {ae }}$, A. Klimentov ${ }^{\mathrm{m}, \mathrm{ab}}$, A.C. König ${ }^{\text {ae }}$, M. Kopal ${ }^{\text {at }}$, V. Koutsenko ${ }^{\text {m,ab }}$, M. Kräber ${ }^{\text {aw }}$, R.W. Kraemer ${ }^{\text {ai }}$, A. Krüger ${ }^{\text {av }}$, A. Kunin ${ }^{\mathrm{m}}$, P. Ladron de Guevara ${ }^{\mathrm{y}}$, I. Laktineh ${ }^{\mathrm{x}}$, G. Landi ${ }^{\mathrm{q}}$, M. Lebeau ${ }^{\mathrm{r}}$, A. Lebedev ${ }^{\mathrm{m}}$, P. Lebrun ${ }^{\mathrm{x}}$,

P. Lecomte ${ }^{\text {aw }}$, P. Lecoq ${ }^{\mathrm{r}}$, P. Le Coultre ${ }^{\text {aw }}$, J.M. Le Goff ${ }^{\mathrm{r}}$, R. Leiste ${ }^{\text {av }}$, M. Levtchenko ${ }^{\text {aa }}$, P. Levtchenko ${ }^{\text {ah }}$, C. Li $^{\text {v }}$, S. Likhoded ${ }^{\text {av }}$, C.H. Lin ${ }^{\text {ar }}$, W.T. Lin ${ }^{\text {ar }}$, F.L. Linde ${ }^{\text {b }}$, L. Lista ${ }^{\text {ac }}$, Z.A. Liu ${ }^{g}$, W. Lohmann ${ }^{\text {av }}$, E. Longo ${ }^{\text {am }}$, Y.S. Lu ${ }^{g}$, C. Luci ${ }^{\text {am }}$, L. Luminari ${ }^{\text {am }}$, W. Lustermann ${ }^{\text {aw }}$, W.G. Ma ${ }^{\mathrm{v}}$, L. Malgeri ${ }^{\mathrm{r}}$, A. Malinin ${ }^{\text {ab }}$, C. Maña ${ }^{\mathrm{y}}$, J. Mans ${ }^{\text {ak }}$, J.P. Martin ${ }^{\mathrm{x}}$, F. Marzano ${ }^{\mathrm{am}}$, K. Mazumdar ${ }^{\mathrm{i}}$, R.R. McNeil ${ }^{\mathrm{f}}$, S. Mele ${ }^{\mathrm{r}, \mathrm{ac}, *}$, L. Merola $^{\mathrm{ac}}$, M. Meschini ${ }^{\mathrm{q}}$, W.J. Metzger ${ }^{\mathrm{ae}}$, A. Mihul ${ }^{\mathrm{k}}$, H. Milcent ${ }^{\mathrm{r}}$, G. Mirabelli ${ }^{a m}$, J. Mnich $^{\mathrm{a}}$, G.B. Mohanty ${ }^{\mathrm{i}}$, G.S. Muanza ${ }^{\mathrm{x}}$, A.J.M. Muijs ${ }^{\mathrm{b}}$, M. Musy ${ }^{\mathrm{am}}, \mathrm{S} \mathrm{Nagy}^{\mathrm{o}}$, S. Natale ${ }^{\text {t }}$, M. Napolitano ${ }^{\text {ac }}$, F. Nessi-Tedaldi ${ }^{\text {aw }}$, S. Nesterov ${ }^{\text {ah }}$, H. Newman ${ }^{\text {af }}$, A. Nisati ${ }^{\text {am }}$, 
T. Novak ${ }^{\text {ae }}$, H. Nowak ${ }^{\text {av }}$, R. Ofierzynski aw ${ }^{\text {aw }}$ G. Organtini ${ }^{\text {am }}$, I. Pal $^{\text {at }}$, C. Palomares ${ }^{\mathrm{y}}$, P. Paolucci $^{\text {ac }}$, R. Paramatti ${ }^{\text {am }}$, G. Passaleva ${ }^{\mathrm{q}}$, S. Patricelli ${ }^{\text {ac }}$, T. Paul ${ }^{\mathrm{j}}$, M. Pauluzzi $^{\mathrm{ag}}$, C. Paus ${ }^{\mathrm{m}}$, F. Pauss ${ }^{\text {aw }}$, M. Pedace ${ }^{a m}$, S. Pensotti $^{\text {aa }}$, D. Perret-Gallix ${ }^{\text {d }}$, D. Piccolo ${ }^{\text {ac }}$, F. Pierella ${ }^{\text {h }}$, M. Pieri ${ }^{\text {ao }}$, M. Pioppi ${ }^{\text {ag }}$, P.A. Piroué ${ }^{\text {ak }}$, E. Pistolesi ${ }^{\text {aa }}$, V. Plyaskin ${ }^{\text {ab }}$, M. Pohl ${ }^{t}$, V. Pojidaev ${ }^{\mathrm{q}}$, J. Pothier $^{\mathrm{r}}$, D. Prokofiev ${ }^{\text {ah }}$, G. Rahal-Callot ${ }^{\text {aw }}$, M.A. Rahaman ${ }^{\mathrm{i}}$, P. Raics ${ }^{\circ}$, N. Raja ${ }^{\mathrm{i}}$, R. Ramelli ${ }^{\text {aw }}$, P.G. Rancoita ${ }^{\text {aa }}$, R. Ranieri ${ }^{\mathrm{q}}$, A. Raspereza ${ }^{\text {av }}$, P. Razis ${ }^{\text {ad }}$, S. Rembeczki ${ }^{z}$, D. Ren ${ }^{\text {aw }}$, M. Rescigno ${ }^{\text {am }}$, S. Reucroft $^{j}$, S. Riemann $^{\text {av }}$, K. Riles ${ }^{\text {c }}$, B.P. Roe ${ }^{c}$, L. Romero ${ }^{y}$, A. Rosca ${ }^{\text {av }}$, C. Rosemann ${ }^{a}$, C. Rosenbleck ${ }^{\mathrm{a}}$, S. Rosier-Lees ${ }^{\mathrm{d}}$, S. Roth ${ }^{\text {a }}$, J.A. Rubio ${ }^{\text {r }}$, G. Ruggiero ${ }^{\text {}}$, H. Rykaczewski ${ }^{\text {aw }}$, A. Sakharov ${ }^{\text {aw }}$, S. Saremi ${ }^{\text {f }}$, S. Sarkar ${ }^{\text {am }}$, J. Salicio ${ }^{r}$, E. Sanchez ${ }^{y}$, C. Schäfer ${ }^{r}$, H. Schopper ${ }^{u}$, D.J. Schotanus ${ }^{\text {ae }}$, C. Sciacca ${ }^{\text {ac }}$, L. Servoli ${ }^{\text {ag }}$, S. Shevchenko af, N. Shivarov ${ }^{\text {ap }}$, V. Shoutko ${ }^{\mathrm{m}}$, E. Shumilov ${ }^{\mathrm{ab}}$, A. Shvorob ${ }^{\text {af }}$, D. Son ${ }^{\text {aq }}$, C. Souga ${ }^{\mathrm{x}}$, P. Spillantini ${ }^{\mathrm{q}}$, M. Steuer ${ }^{\mathrm{m}}$, D.P. Stickland ${ }^{\text {ak }}$, B. Stoyanov ${ }^{\text {ap }}$, A. Straessner ${ }^{\mathrm{t}}$, K. Sudhakar ${ }^{\mathrm{i}}$, G. Sultanov ${ }^{\text {ap }}$, L.Z. Sun ${ }^{\text {v }, ~ S . ~ S u s h k o v ~}{ }^{\text {a }}$, H. Suter ${ }^{\text {aw }}$, J.D. Swain ${ }^{\text {j }, ~ Z . ~ S z i l l a s i ~}{ }^{\text {z,2 }}$, X.W. Tang ${ }^{\mathrm{g}}$, P. $\operatorname{Tarjan}^{\mathrm{o}}$, L. Tauscher ${ }^{\mathrm{e}}$, L. Taylor $^{\mathrm{j}}$, B. Tellili ${ }^{\mathrm{x}}$, D. Teyssier ${ }^{\mathrm{x}}$, C. Timmermans ${ }^{\mathrm{ae}}$, Samuel C.C. Ting ${ }^{\mathrm{m}}$, S.M. Ting ${ }^{\mathrm{m}}$, S.C. Tonwar ${ }^{\mathrm{i}}$, J. Tóth ${ }^{1}$, C. Tully ${ }^{\mathrm{ak}}{ }$, K.L. Tung ${ }^{\mathrm{g}}$, J. Ulbricht ${ }^{\mathrm{aw}}$, E. Valente ${ }^{\mathrm{am}}$, R.T. Van de Walle ${ }^{\mathrm{ae}}$, R. Vasquez ${ }^{\text {at }}$, G. Vesztergombi ${ }^{1}$, I. Vetlitsky ${ }^{\mathrm{ab}}$, G. Viertel $^{\text {aw }}$, M. Vivargent ${ }^{\mathrm{d}}$, S. Vlachos ${ }^{\mathrm{e}}$, I. Vodopianov ${ }^{\mathrm{z}}$, H. Vogel $^{\text {ai }}, \mathrm{H}$. Vogt ${ }^{\mathrm{av}}$, I. Vorobiev ${ }^{\mathrm{ai}, \mathrm{ab}}$, A.A. Vorobyov ${ }^{\text {ah }}$, M. Wadhwa ${ }^{\mathrm{e}}$, Q. Wang ${ }^{\text {ae }}$, X.L. Wang ${ }^{\mathrm{v}}$, Z.M. Wang ${ }^{\mathrm{v}}$, M. Weber ${ }^{\mathrm{r}}$, S. Wynhoff $^{\text {ak, }}$, $^{\text {, }}$, L. Xia $^{\text {af }}$, Z.Z. Xu ${ }^{\text {}}$, J. Yamamoto ${ }^{\text {c }}$, B.Z. Yang ${ }^{\text {v }, \text { C.G. Yang }}{ }^{\mathrm{g}}$, H.J. Yang ${ }^{\mathrm{c}}$, M. Yang ${ }^{\mathrm{g}}$, S.C. Yeh $^{\text {as }}$, An. Zalite ${ }^{\text {ah }}$, Yu. Zalite ${ }^{\text {ah }}$, Z.P. Zhang ${ }^{\text {v }}$, J. Zhao ${ }^{\text {, }}$, G.Y. Zhu ${ }^{\mathrm{g}}$, R. Y. Zhu ${ }^{\text {af }}$, H.L. Zhuang ${ }^{\mathrm{g}}$, A. Zichichi ${ }^{\text {h,r,s }}$, B. Zimmermann ${ }^{\text {aw }}$, M. Zöller ${ }^{\text {a }}$

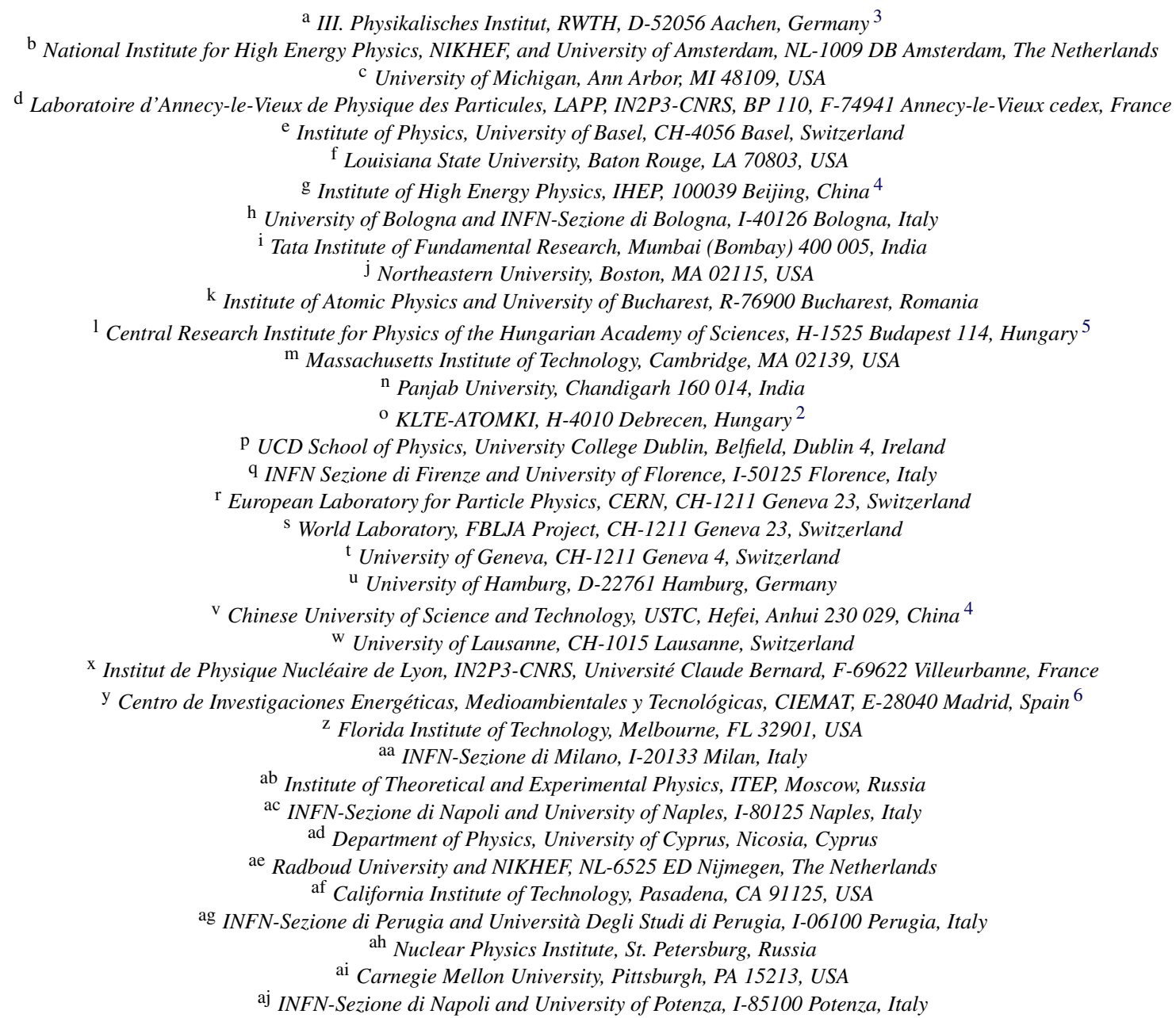




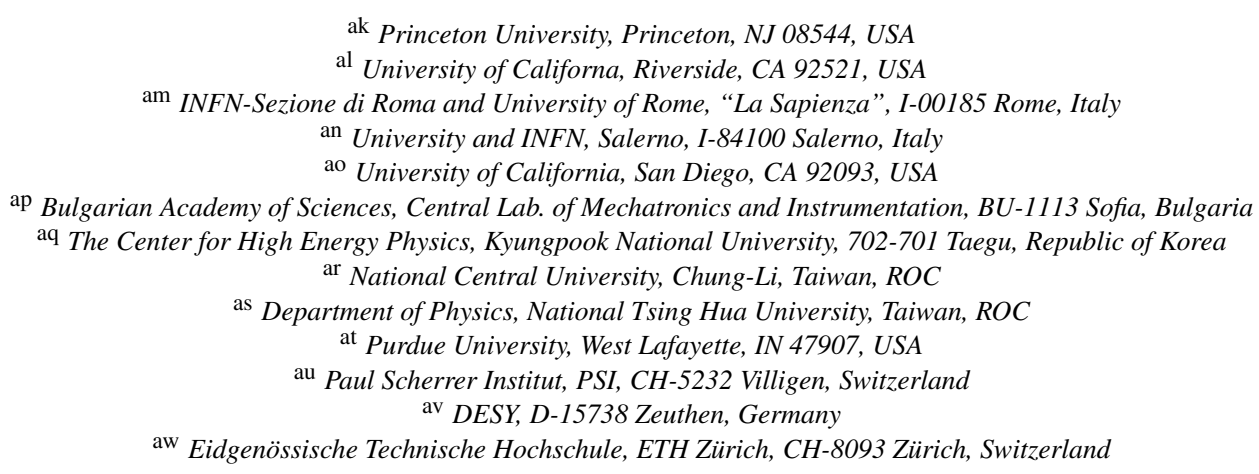

Received 12 December 2005; received in revised form 30 March 2006; accepted 4 May 2006

Available online 15 May 2006

Editor: L. Rolandi

\begin{abstract}
The reactions $\gamma \gamma \rightarrow \pi^{+} \pi^{-} \pi^{+} \pi^{-}$and $\gamma \gamma \rightarrow \pi^{+} \pi^{0} \pi^{-} \pi^{0}$ are studied with the L3 detector at LEP in a data sample collected at centre-of-mass energies from $161 \mathrm{GeV}$ to $209 \mathrm{GeV}$ with a total integrated luminosity of $698 \mathrm{pb}^{-1}$. A spin-parity-helicity analysis of the $\rho^{0} \rho^{0}$ and $\rho^{+} \rho^{-}$systems for two-photon centre-of-mass energies between 1 and $3 \mathrm{GeV}$ shows the dominance of the spin-parity state $2^{+}$with helicity 2 . The contribution of $0^{+}$and $0^{-}$spin-parity states is also observed, whereas contributions of $2^{-}$states and of a state with spin-parity $2^{+}$and zero helicity are found to be negligible.
\end{abstract}

(c) 2006 Elsevier B.V. All rights reserved.

\section{Introduction}

Several experiments have observed a large cross section near threshold for the reaction $\gamma \gamma \rightarrow \rho^{0} \rho^{0}$ [1-3]. In contrast, the corresponding cross section for the isospin-related reaction $\gamma \gamma \rightarrow \rho^{+} \rho^{-}$was shown to be small [4,5]. The first spin-parityhelicity analysis of the reaction $\gamma \gamma \rightarrow \pi^{+} \pi^{-} \pi^{+} \pi^{-}$was carried out by the TASSO Collaboration [2] by studying angular correlations. The data sample consisted of 1722 events for twophoton centre-of-mass energies $1.2 \mathrm{GeV}<W_{\gamma \gamma}<2.0 \mathrm{GeV}$. A spin-parity-helicity analysis with higher statistics was performed by the ARGUS Collaboration [3] with 5181 events in the region $1.1 \mathrm{GeV}<W_{\gamma \gamma}<2.3 \mathrm{GeV}$. Both collaborations used similar models and observed the dominance of $\rho^{0} \rho^{0}$ states with spin-parity $J^{P}=2^{+}$and $0^{+}$. The contribution of negativeparity states was found to be negligible.

A number of theoretical models [6] were proposed to interpret these experimental results. In a $t$-channel factorization approach [7], the $\gamma \gamma \rightarrow \rho^{0} \rho^{0}$ cross section is related to photo-

\footnotetext{
* Corresponding author.

1 Also supported by CONICET and Universidad Nacional de La Plata, CC 67, 1900 La Plata, Argentina.

2 Also supported by the Hungarian OTKA fund under contract number T026178

3 Supported by the German Bundesministerium für Bildung, Wissenschaft, Forschung und Technologie.

4 Supported by the National Natural Science Foundation of China.

5 Supported by the Hungarian OTKA fund under contract numbers T019181, F023259 and T037350.

${ }^{6}$ Supported also by the Comisión Interministerial de Ciencia y Tecnología.

Deceased.
}

production and hadronic cross sections at low energies. This model leads to the interpretation of the broad enhancement in the $\gamma \gamma \rightarrow \rho^{0} \rho^{0}$ cross section around $1.6 \mathrm{GeV}$ as a threshold behaviour due to Regge exchange. Other models suggest an $s$-channel $\rho^{0} \rho^{0}$ resonance [8,9], either a normal $q \bar{q}$ state or a four-quark $q q \bar{q} \bar{q}$ bound state. In four-quark models, isoscalar and isotensor resonances interfere destructively to suppress the $\gamma \gamma \rightarrow \rho^{+} \rho^{-}$signal and constructively to describe the $\gamma \gamma \rightarrow$ $\rho^{0} \rho^{0}$ cross section. The proposed models differ substantially in the predicted cross section for the production of other vector mesons such as $\gamma \gamma \rightarrow \rho^{0} \omega$ and $\gamma \gamma \rightarrow \phi \phi$.

This Letter presents the results of a spin-parity-helicity analysis of the reactions $\gamma \gamma \rightarrow \pi^{+} \pi^{-} \pi^{+} \pi^{-}$and $\gamma \gamma \rightarrow$ $\pi^{+} \pi^{0} \pi^{-} \pi^{0}$ in data collected by the L3 detector [10] at LEP, using the same technique as TASSO and ARGUS. The data samples consist of $7.5 \times 10^{4}$ events for the $\mathrm{e}^{+} \mathrm{e}^{-} \rightarrow \mathrm{e}^{+} \mathrm{e}^{-} \pi^{+} \pi^{-}$ $\pi^{+} \pi^{-}$channel and $7.5 \times 10^{3}$ events for the $\mathrm{e}^{+} \mathrm{e}^{-} \rightarrow \mathrm{e}^{+} \mathrm{e}^{-} \pi^{+}$ $\pi^{0} \pi^{-} \pi^{0}$ channel. These data are selected in the region of quasireal photons with a maximum virtuality of $Q^{2} \simeq 0.02 \mathrm{GeV}^{2}$. The $\gamma \gamma \rightarrow \rho^{0} \rho^{0}$ and $\gamma \gamma \rightarrow \rho^{+} \rho^{-}$cross sections obtained in this analysis are compared to the high-virtuality [11,12] and mid-virtuality $[13,14]$ data obtained with the same detector.

\section{Data and Monte Carlo samples}

The two-photon production of a $\rho$-pair, $\gamma \gamma \rightarrow \rho^{0} \rho^{0}$ or $\gamma \gamma \rightarrow \rho^{+} \rho^{-}$, is observed via the reactions $\mathrm{e}^{+} \mathrm{e}^{-} \rightarrow \mathrm{e}^{+} \mathrm{e}^{-} \pi^{+}$ $\pi^{-} \pi^{+} \pi^{-}$or $\mathrm{e}^{+} \mathrm{e}^{-} \rightarrow \mathrm{e}^{+} \mathrm{e}^{-} \pi^{+} \pi^{0} \pi^{-} \pi^{0}$, respectively. Detection of the scattered leptons is not required. The data were collected with the $\mathrm{L} 3$ detector at $\mathrm{e}^{+} \mathrm{e}^{-}$centre-of-mass ener- 

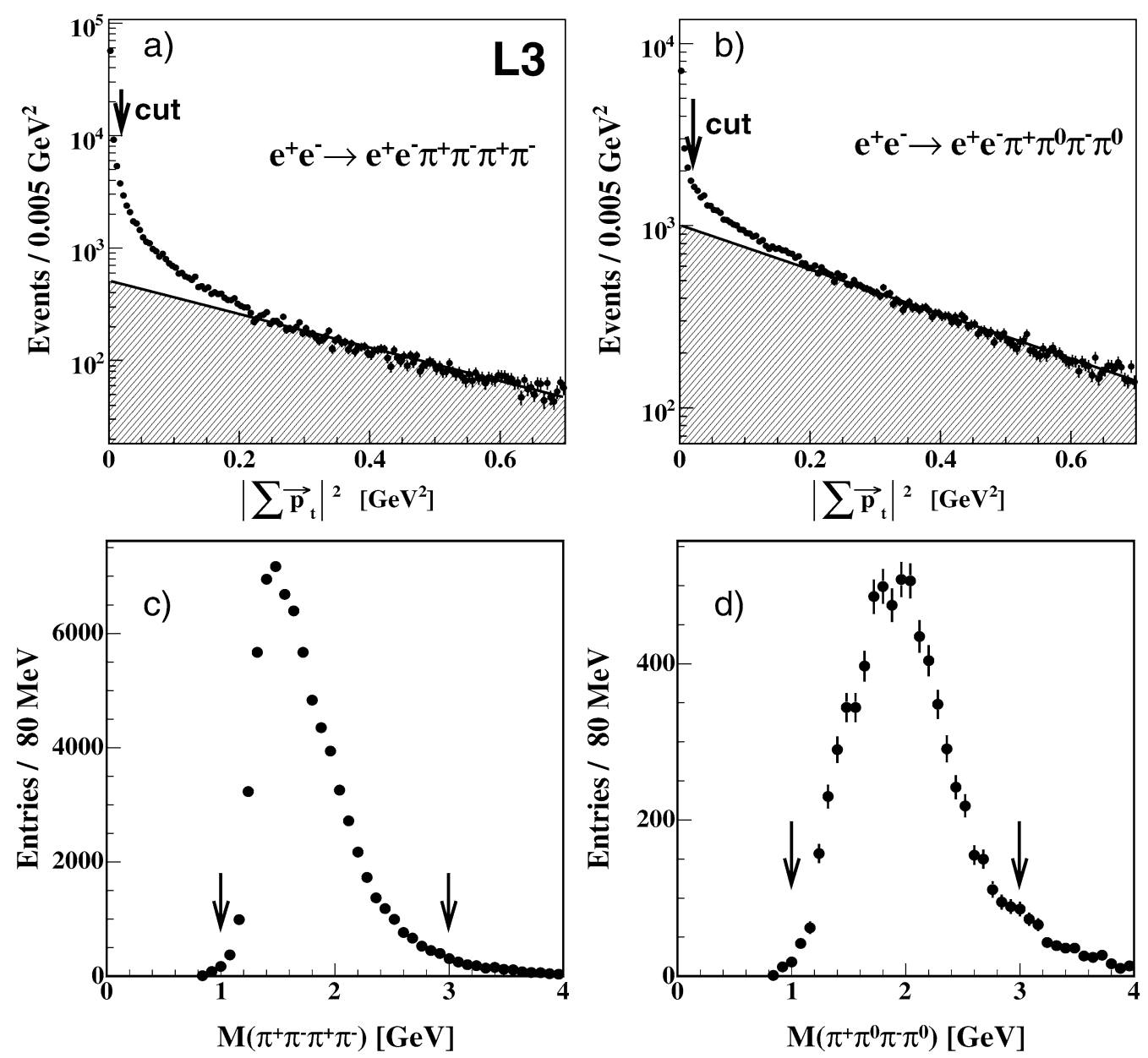

Fig. 1. Distributions of $\left|\Sigma \vec{p}_{\mathrm{t}}\right|^{2}$ for (a) $\mathrm{e}^{+} \mathrm{e}^{-} \rightarrow \mathrm{e}^{+} \mathrm{e}^{-} \pi^{+} \pi^{-} \pi^{+} \pi^{-}$and (b) $\mathrm{e}^{+} \mathrm{e}^{-} \rightarrow \mathrm{e}^{+} \mathrm{e}^{-} \pi^{+} \pi^{0} \pi^{-} \pi^{0}$ events. The hatched areas represent the estimated non-exclusive backgrounds. The cut values are shown by the arrows. Distributions of the four-pion mass for (c) $\mathrm{e}^{+} \mathrm{e}^{-} \rightarrow \mathrm{e}^{+} \mathrm{e}^{-} \pi^{+} \pi^{-} \pi^{+} \pi^{-}$and (d) $\mathrm{e}^{+} \mathrm{e}^{-} \rightarrow \mathrm{e}^{+} \mathrm{e}^{-} \pi^{+} \pi^{0} \pi^{-} \pi^{0}$ events. Only events within the region indicated by the arrows are further analysed.

gies $\sqrt{s}=161-209 \mathrm{GeV}$, with a total integrated luminosity $\mathcal{L}_{\mathrm{e}^{+} \mathrm{e}^{-}}=697.7 \mathrm{pb}^{-1}$ and an average centre-of-mass energy of $196 \mathrm{GeV}$. The analysis described in this Letter is mainly based on the central tracking system and the electromagnetic calorimeter.

Four-pion Monte Carlo events are generated with the EGPC [15] program. The four-momentum of the two-photon system is distributed according to the transverse two-photon luminosity function [16]. The pion four-momentum vectors are generated using four-particle phase space. The events are then passed through the L3 detector simulation, which uses the GEANT [17] and GEISHA [18] programs, and are reconstructed following the same procedure as used for the data.

\section{Event selection}

The events are collected by two charged-track triggers. The first trigger [19] requires at least two wide-angle tracks, backto-back within $\pm 41^{\circ}$ in the plane transverse to the beam. The second trigger [20] is based on a neural network which was trained to select low-multiplicity events while rejecting beamgas and beam-wall background.

Events are selected by requiring:
- Four charged tracks for the $\mathrm{e}^{+} \mathrm{e}^{-} \rightarrow \mathrm{e}^{+} \mathrm{e}^{-} \pi^{+} \pi^{-} \pi^{+} \pi^{-}$ reaction and two charged tracks for the $\mathrm{e}^{+} \mathrm{e}^{-} \rightarrow \mathrm{e}^{+} \mathrm{e}^{-} \pi^{+}$ $\pi^{0} \pi^{-} \pi^{0}$ reaction, with a net charge of zero in each case. A track is required to have: more than 12 hits, with at least $60 \%$ of possible hits, a transverse momentum, $p_{\mathrm{t}}$, greater than $100 \mathrm{MeV}$ and a distance of closest approach to the interaction vertex in the transverse plane less than $2 \mathrm{~mm}$.

- No photons for the $\gamma \gamma \rightarrow \pi^{+} \pi^{-} \pi^{+} \pi^{-}$reaction and four isolated clusters in the electromagnetic calorimeter for the $\gamma \gamma \rightarrow \pi^{+} \pi^{0} \pi^{-} \pi^{0}$ reaction. A photon is defined as an isolated shower in the electromagnetic calorimeter consisting of at least two adjacent crystals with an energy greater than $100 \mathrm{MeV}$ and with no charged track within 200 mrad.

- An energy loss $\mathrm{d} E / \mathrm{d} x$ in the tracking chamber corresponding to the hypothesis that all the charged particles are pions, with a confidence level greater than $6 \%$.

- Two pairs of photons each with a good fit to the $\pi^{0}$ decay hypothesis for the $\pi^{+} \pi^{0} \pi^{-} \pi^{0}$ final state.

To suppress the background from non-exclusive events, the overall transverse momentum of the event, $\left|\Sigma \vec{p}_{\mathrm{t}}\right|^{2}$, must be less than $0.02 \mathrm{GeV}^{2}$, as shown in Figs. 1(a) and (b). The resulting samples consist of 74859 and 7535 events for the 

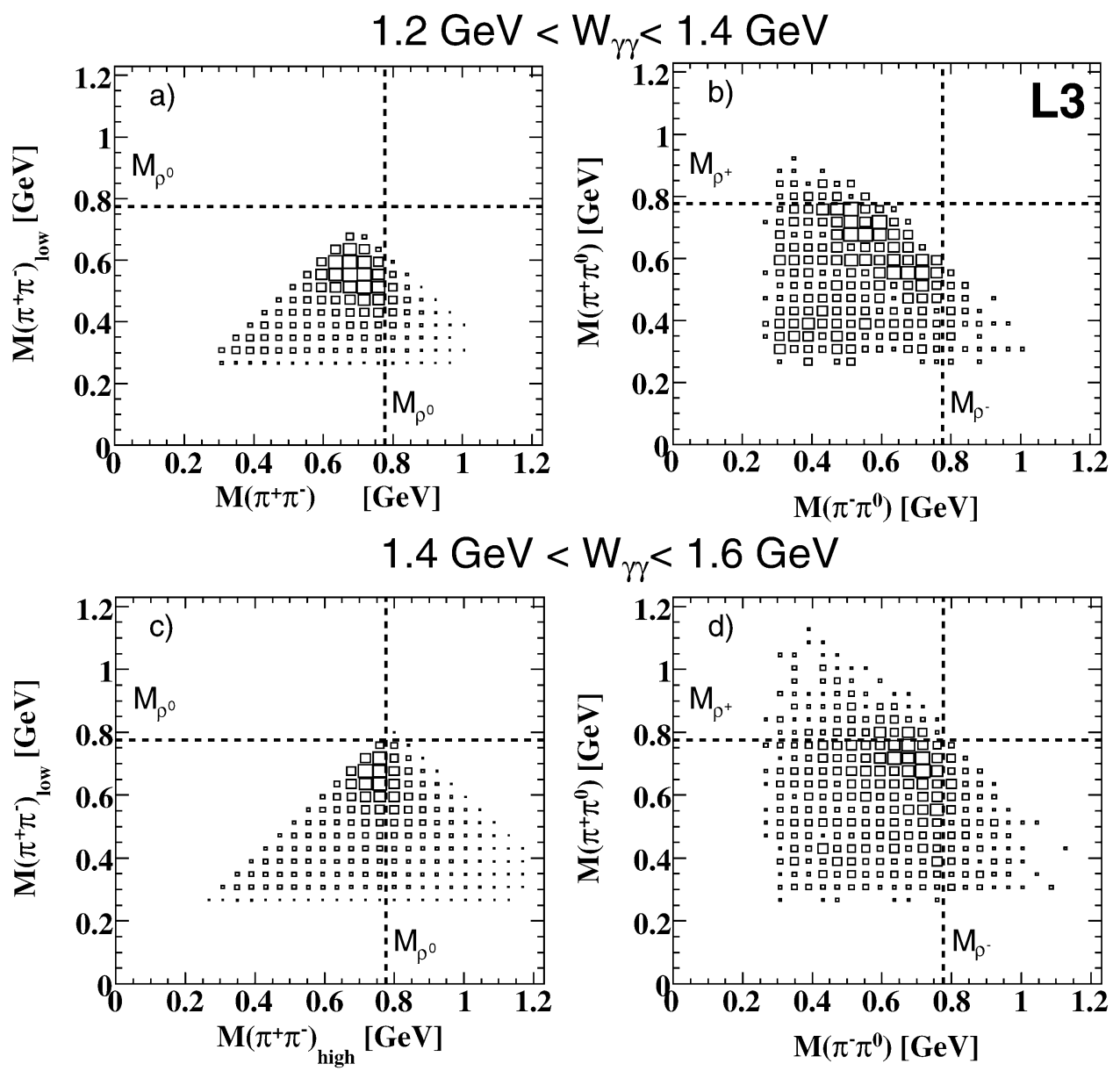

$1.6 \mathrm{GeV}<\mathrm{W}_{\gamma \gamma}<3.0 \mathrm{GeV}$
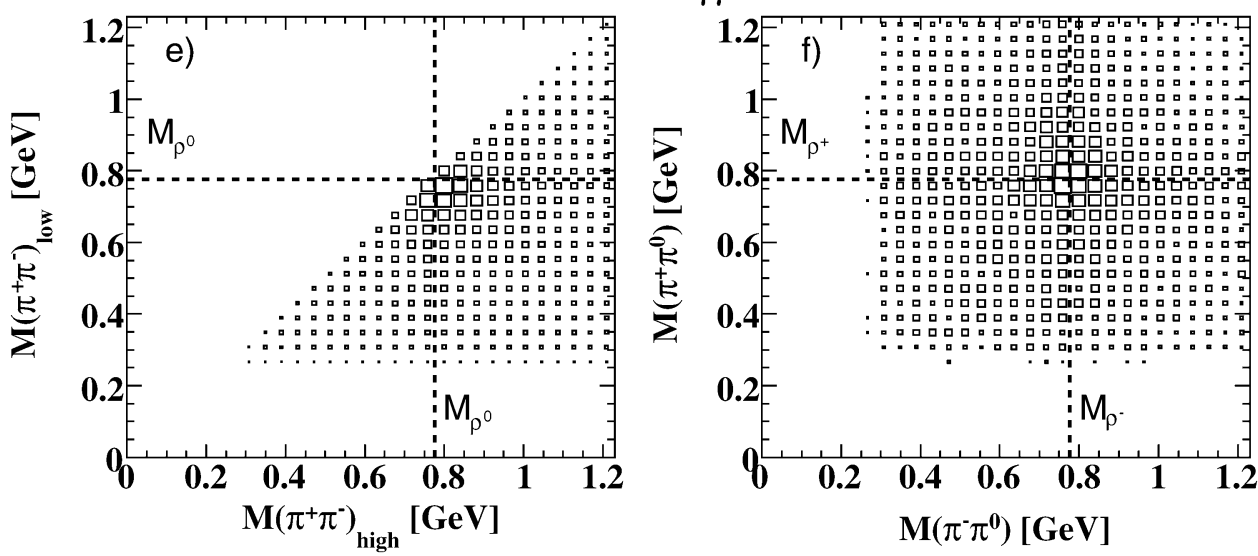

Fig. 2. Two-dimensional distributions of two-pion masses in three different $W_{\gamma \gamma}$ regions. For (a), (c) and (e) the $\pi^{+} \pi^{-}$combinations from the $\pi^{+} \pi^{-} \pi^{+} \pi^{-}$ final-state are shown as low-mass vs. high-mass, with two entries per event. In (b), (d) and (f) the $\pi^{+} \pi^{0}$ vs. $\pi^{-} \pi^{0}$ combinations from the $\pi^{+} \pi^{0} \pi^{-} \pi^{0}$ final-state are shown, with two entries per event. The dotted lines indicate the nominal mass value of the $\rho$ meson.

$\mathrm{e}^{+} \mathrm{e}^{-} \rightarrow \mathrm{e}^{+} \mathrm{e}^{-} \pi^{+} \pi^{-} \pi^{+} \pi^{-}$and $\mathrm{e}^{+} \mathrm{e}^{-} \rightarrow \mathrm{e}^{+} \mathrm{e}^{-} \pi^{+} \pi^{0} \pi^{-} \pi^{0}$ reactions, respectively.

The distributions of the four-pion mass, equal to $W_{\gamma \gamma}$ for exclusive events, are shown in Figs. 1(c) and (d). The mass resolution is estimated to be $48 \mathrm{MeV}$ for the $\pi^{+} \pi^{-} \pi^{+} \pi^{-}$and $63 \mathrm{MeV}$ for the $\pi^{+} \pi^{0} \pi^{-} \pi^{0}$ final states. More than $90 \%$ of the events lie in the region $1.0 \mathrm{GeV} \leqslant W_{\gamma \gamma} \leqslant 3.0 \mathrm{GeV}$, where the spin-parity-helicity analysis is performed.
The background is dominated by higher-multiplicity final states produced in two-photon interactions which are only partially reconstructed. The expected contribution from annihilation events is negligible. As presented in Figs. 1(a) and (b), the distribution of $\left|\Sigma \vec{p}_{\mathrm{t}}\right|^{2}$ for non-exclusive final states has an exponential form, which is estimated from the data in the high $\left|\Sigma \vec{p}_{\mathrm{t}}\right|^{2}$ region, $0.2 \mathrm{GeV}^{2} \leqslant\left|\Sigma \vec{p}_{\mathrm{t}}\right|^{2} \leqslant 0.8 \mathrm{GeV}^{2}$. Extrapolating this exponential to the signal region, $\left|\Sigma \vec{p}_{\mathrm{t}}\right|^{2}<0.02 \mathrm{GeV}^{2}$, the 
Table 1

Cross section measurements and fit results for $\gamma \gamma \rightarrow \pi^{+} \pi^{-} \pi^{+} \pi^{-}$for different $W_{\gamma \gamma}$ intervals. $N$ is the number of events in a bin, $\int \mathrm{d} \mathcal{L}_{\gamma \gamma}$ the two-photon luminosity function, $\varepsilon_{\operatorname{trg}}$ the trigger efficiency and $\varepsilon$ the selection efficiency. The cross sections for the background, $4 \pi$, and for the different spin-helicity waves are given, along with the total $\gamma \gamma \rightarrow \rho^{0} \rho^{0}$ cross section. A double dash indicates that no significant contribution to the fit is observed. The first uncertainties are statistical, the second systematic

\begin{tabular}{|c|c|c|c|c|c|c|c|c|c|}
\hline$W_{\gamma \gamma}[\mathrm{GeV}]$ & $N$ & $\int \mathrm{d} \mathcal{L}_{\gamma \gamma}\left[10^{-3}\right]$ & $\varepsilon_{\operatorname{trg}}[\%]$ & $\varepsilon[\%]$ & $4 \pi[\mathrm{nb}]$ & $0^{+}[\mathrm{nb}]$ & $0^{-}[\mathrm{nb}]$ & $\left(2^{+}, 2\right)[\mathrm{nb}]$ & $\sigma_{\text {tot }}\left(\gamma \gamma \rightarrow \rho^{0} \rho^{0}\right)[\mathrm{nb}]$ \\
\hline $1.00-1.10$ & 376 & 4.06 & 94.2 & 1.8 & $3.8 \pm 0.7 \pm 0.1$ & $0.6 \pm 0.4 \pm 0.1$ & - & $1.4 \pm 0.5 \pm 0.1$ & $2.1 \pm 0.7 \pm 0.1$ \\
\hline $1.10-1.20$ & 1099 & 3.58 & 94.2 & 2.7 & $3.7 \pm 0.7 \pm 0.1$ & $0.7 \pm 0.4 \pm 0.2$ & - & $6.2 \pm 0.6 \pm 0.2$ & $6.9 \pm 0.7 \pm 0.2$ \\
\hline $1.20-1.30$ & 4513 & 3.20 & 95.3 & 3.5 & $5.3 \pm 1.0 \pm 0.4$ & $5.1 \pm 1.1 \pm 0.4$ & $0.8 \pm 0.6 \pm 0.1$ & $23.2 \pm 1.4 \pm 1.8$ & $29.1 \pm 1.9 \pm 2.2$ \\
\hline $1.30-1.40$ & 7717 & 2.87 & 95.3 & 4.2 & $16.3 \pm 1.3 \pm 1.0$ & $7.7 \pm 1.3 \pm 0.5$ & $1.4 \pm 0.7 \pm 0.1$ & $30.5 \pm 1.6 \pm 1.9$ & $39.6 \pm 2.2 \pm 2.5$ \\
\hline $1.40-1.50$ & 9084 & 2.60 & 95.3 & 4.8 & $18.2 \pm 1.2 \pm 1.0$ & $13.7 \pm 1.5 \pm 0.8$ & $1.8 \pm 0.7 \pm 0.1$ & $31.7 \pm 1.7 \pm 1.8$ & $47.1 \pm 2.4 \pm 2.7$ \\
\hline $1.50-1.60$ & 8397 & 2.37 & 95.8 & 5.4 & $19.8 \pm 1.2 \pm 2.2$ & $9.8 \pm 1.5 \pm 1.1$ & $4.3 \pm 0.9 \pm 0.5$ & $34.5 \pm 2.0 \pm 3.8$ & $48.6 \pm 2.6 \pm 5.3$ \\
\hline $1.60-1.70$ & 7910 & 2.17 & 95.8 & 5.9 & $19.3 \pm 1.2 \pm 2.1$ & $5.9 \pm 1.3 \pm 0.7$ & $2.1 \pm 0.7 \pm 0.2$ & $35.6 \pm 1.8 \pm 3.9$ & $43.7 \pm 2.4 \pm 4.8$ \\
\hline $1.70-1.80$ & 6671 & 2.00 & 96.2 & 6.3 & $19.0 \pm 1.2 \pm 1.0$ & $7.7 \pm 1.3 \pm 0.4$ & $4.8 \pm 0.8 \pm 0.2$ & $26.4 \pm 1.6 \pm 1.3$ & $39.0 \pm 2.2 \pm 2.0$ \\
\hline $1.80-1.90$ & 5643 & 1.85 & 96.2 & 6.7 & $20.7 \pm 1.3 \pm 1.7$ & $4.9 \pm 1.1 \pm 0.4$ & $7.4 \pm 0.9 \pm 0.6$ & $23.6 \pm 1.5 \pm 1.9$ & $35.9 \pm 2.1 \pm 2.9$ \\
\hline $1.90-2.00$ & 4965 & 1.72 & 96.2 & 7.1 & $27.8 \pm 1.6 \pm 3.3$ & $7.3 \pm 1.2 \pm 0.9$ & $4.8 \pm 0.8 \pm 0.6$ & $15.0 \pm 1.3 \pm 1.8$ & $27.1 \pm 1.9 \pm 3.2$ \\
\hline $2.00-2.10$ & 4004 & 1.60 & 96.4 & 7.4 & $26.2 \pm 1.6 \pm 6.0$ & $9.5 \pm 1.3 \pm 2.2$ & $4.0 \pm 0.7 \pm 0.9$ & $7.9 \pm 1.1 \pm 1.8$ & $21.3 \pm 1.8 \pm 4.9$ \\
\hline $2.10-2.20$ & 3118 & 1.49 & 96.4 & 7.7 & $24.4 \pm 1.5 \pm 8.9$ & $4.8 \pm 1.0 \pm 1.8$ & $2.0 \pm 0.5 \pm 0.7$ & $7.2 \pm 1.0 \pm 2.6$ & $13.9 \pm 1.5 \pm 5.1$ \\
\hline $2.20-2.30$ & 2366 & 1.40 & 96.2 & 7.9 & $21.0 \pm 1.4 \pm 8.5$ & $2.2 \pm 0.7 \pm 0.9$ & $1.8 \pm 0.5 \pm 0.7$ & $5.2 \pm 0.9 \pm 2.1$ & $9.2 \pm 1.3 \pm 3.7$ \\
\hline $2.30-2.40$ & 1763 & 1.31 & 96.2 & 8.1 & $17.3 \pm 1.3 \pm 9.7$ & $1.6 \pm 0.6 \pm 0.9$ & $2.6 \pm 0.6 \pm 1.5$ & $2.6 \pm 0.7 \pm 1.4$ & $6.8 \pm 1.1 \pm 3.8$ \\
\hline $2.40-2.50$ & 1450 & 1.24 & 96.2 & 8.4 & $15.0 \pm 1.1 \pm 8.9$ & $2.0 \pm 0.6 \pm 1.2$ & $1.7 \pm 0.5 \pm 1.0$ & $1.4 \pm 0.7 \pm 0.8$ & $5.1 \pm 1.0 \pm 3.0$ \\
\hline $2.50-2.60$ & 1137 & 1.17 & 95.8 & 8.6 & $12.1 \pm 1.1 \pm 7.3$ & $2.0 \pm 0.7 \pm 1.2$ & $1.0 \pm 0.4 \pm 0.6$ & $2.1 \pm 0.8 \pm 1.2$ & $5.1 \pm 1.1 \pm 3.1$ \\
\hline $2.60-2.70$ & 878 & 1.10 & 95.8 & 8.8 & $10.7 \pm 1.0 \pm 6.8$ & $1.4 \pm 0.5 \pm 0.9$ & $1.5 \pm 0.4 \pm 1.0$ & - & $2.9 \pm 0.6 \pm 1.9$ \\
\hline $2.70-2.80$ & 672 & 1.05 & 96.5 & 8.9 & $8.5 \pm 0.9 \pm 2.3$ & $1.1 \pm 0.3 \pm 0.3$ & - & $1.1 \pm 0.3 \pm 0.3$ & $2.2 \pm 0.5 \pm 0.6$ \\
\hline $2.80-2.90$ & 545 & 0.99 & 96.5 & 9.1 & $7.3 \pm 0.8 \pm 1.1$ & $0.7 \pm 0.2 \pm 0.1$ & - & $1.4 \pm 0.3 \pm 0.2$ & $2.1 \pm 0.4 \pm 0.3$ \\
\hline $2.90-3.00$ & 467 & 0.94 & 96.5 & 9.3 & $6.4 \pm 0.8 \pm 0.1$ & $1.5 \pm 0.5 \pm 0.1$ & - & - & $1.7 \pm 0.6 \pm 0.1$ \\
\hline $1.00-3.00$ & 72775 & 38.72 & - & - & $14.2 \pm 1.1 \pm 2.7$ & $4.9 \pm 0.9 \pm 0.6$ & $2.0 \pm 0.5 \pm 0.3$ & $15.4 \pm 1.1 \pm 1.5$ & $22.3 \pm 1.6 \pm 2.5$ \\
\hline
\end{tabular}

Table 2

Cross section measurement and fit results for $\gamma \gamma \rightarrow \pi^{+} \pi^{0} \pi^{-} \pi^{0}$ for different $W_{\gamma \gamma}$ intervals. $N$ is the number of events in a bin, $\int \mathrm{d} \mathcal{L}_{\gamma \gamma}$ the two-photon luminosity function, $\varepsilon_{\text {trg }}$ the trigger efficiency and $\varepsilon$ the selection efficiency. The cross sections for the background, $4 \pi$, and for the different spin-helicity waves are given together with the total $\gamma \gamma \rightarrow \rho^{+} \rho^{-}$cross section. A double dash indicates that no significant contribution to the fit is observed. The first uncertainties are statistical, the second systematic

\begin{tabular}{|c|c|c|c|c|c|c|c|c|c|}
\hline$W_{\gamma \gamma}[\mathrm{GeV}]$ & $N$ & $\int \mathrm{d} \mathcal{L}_{\gamma \gamma}\left[10^{-3}\right]$ & $\varepsilon_{\operatorname{trg}}[\%]$ & $\varepsilon[\%]$ & $4 \pi[\mathrm{nb}]$ & $0^{+}[\mathrm{nb}]$ & $0^{-}[\mathrm{nb}]$ & $\left(2^{+}, 2\right)[\mathrm{nb}]$ & $\sigma_{\text {tot }}\left(\gamma \gamma \rightarrow \rho^{+} \rho^{-}\right)[\mathrm{nb}]$ \\
\hline $1.00-1.20$ & 111 & 7.64 & 66.4 & 0.3 & $7.6 \pm 2.2 \pm 1.1$ & $0.6 \pm 1.1 \pm 0.1$ & $0.6 \pm 0.8 \pm 0.1$ & $1.0 \pm 1.2 \pm 0.1$ & $2.2 \pm 1.8 \pm 0.3$ \\
\hline $1.20-1.40$ & 526 & 6.07 & 63.5 & 0.5 & $20.1 \pm 3.3 \pm 2.8$ & $3.7 \pm 2.7 \pm 0.5$ & $1.8 \pm 1.3 \pm 0.2$ & $7.9 \pm 2.7 \pm 1.1$ & $13.4 \pm 4.0 \pm 1.8$ \\
\hline $1.40-1.60$ & 839 & 4.97 & 65.0 & 0.8 & $30.7 \pm 3.4 \pm 5.5$ & $1.5 \pm 2.1 \pm 0.3$ & $4.5 \pm 1.3 \pm 0.8$ & $5.0 \pm 2.0 \pm 0.9$ & $10.9 \pm 3.2 \pm 2.0$ \\
\hline $1.60-1.80$ & 1160 & 4.17 & 65.0 & 1.0 & $30.8 \pm 3.5 \pm 5.5$ & $4.8 \pm 2.2 \pm 0.9$ & $1.4 \pm 1.0 \pm 0.3$ & $12.3 \pm 2.3 \pm 2.2$ & $18.6 \pm 3.3 \pm 3.3$ \\
\hline $1.80-2.00$ & 1205 & 3.56 & 59.9 & 1.3 & $32.2 \pm 3.8 \pm 8.6$ & $3.8 \pm 2.3 \pm 1.0$ & $2.9 \pm 1.5 \pm 0.8$ & $19.2 \pm 3.0 \pm 5.1$ & $25.9 \pm 4.1 \pm 6.9$ \\
\hline $2.00-2.20$ & 1161 & 3.09 & 63.4 & 1.6 & $34.0 \pm 3.7 \pm 8.3$ & $8.5 \pm 2.2 \pm 2.1$ & - & $9.1 \pm 2.1 \pm 2.2$ & $17.7 \pm 3.2 \pm 4.3$ \\
\hline $2.20-2.40$ & 823 & 2.71 & 64.4 & 1.8 & $27.9 \pm 3.3 \pm 12$ & $2.6 \pm 1.4 \pm 1.1$ & $2.6 \pm 1.2 \pm 1.1$ & $3.5 \pm 1.6 \pm 1.5$ & $8.6 \pm 2.5 \pm 3.7$ \\
\hline $2.40-2.60$ & 540 & 2.41 & 62.8 & 2.1 & $17.2 \pm 2.5 \pm 7.4$ & $1.7 \pm 1.1 \pm 0.7$ & $1.0 \pm 0.7 \pm 0.4$ & $2.7 \pm 1.3 \pm 1.1$ & $5.4 \pm 1.8 \pm 2.3$ \\
\hline $2.60-2.80$ & 336 & 2.15 & 62.8 & 2.3 & $12.0 \pm 2.0 \pm 3.8$ & $1.6 \pm 0.9 \pm 0.5$ & $2.4 \pm 1.1 \pm 0.8$ & - & $4.3 \pm 1.8 \pm 1.4$ \\
\hline $2.80-3.00$ & 231 & 1.94 & 68.7 & 2.6 & $7.2 \pm 1.4 \pm 1.4$ & $1.3 \pm 0.6 \pm 0.3$ & - & $1.4 \pm 0.6 \pm 0.3$ & $2.7 \pm 0.8 \pm 0.5$ \\
\hline $1.00-3.00$ & 6932 & 38.72 & - & - & $21.7 \pm 3.0 \pm 5.8$ & $2.9 \pm 1.8 \pm 0.7$ & $1.8 \pm 1.0 \pm 0.5$ & $6.4 \pm 1.9 \pm 1.5$ & $11.0 \pm 2.8 \pm 2.7$ \\
\hline
\end{tabular}

backgrounds for the $\pi^{+} \pi^{-} \pi^{+} \pi^{-}$and $\pi^{+} \pi^{0} \pi^{-} \pi^{0}$ final states are estimated to be $2.5 \%$ and $4 \%$, respectively.

Figs. 2(a), (c) and (e) show the two-dimensional distributions of the masses of $\pi^{+} \pi^{-}$combinations for the selected $\pi^{+} \pi^{-} \pi^{+} \pi^{-}$events in different $W_{\gamma \gamma}$ regions. There are two entries per event, displayed by ordering the two masses of each entry. Figs. 2(b), (d) and (f) show the $\pi^{+} \pi^{0}$ and $\pi^{-} \pi^{0}$ mass combinations for the $\pi^{+} \pi^{0} \pi^{-} \pi^{0}$ channel with two entries per event.

The two-pion mass resolution is estimated from Monte Carlo simulation to be $25 \mathrm{MeV}$ for both the $\pi^{+} \pi^{-}$and $\pi^{ \pm} \pi^{0}$ cases. The $\pi^{+} \pi^{-}$and $\pi^{ \pm} \pi^{0}$ combinations shown in Fig. 2 present clear evidence of $\rho \rho$ production. For $W_{\gamma \gamma}<1.6 \mathrm{GeV}$, the $\rho$ signal is distorted by threshold effects. As $W_{\gamma \gamma}$ increases, the $\rho$ signal shifts to its nominal mass value, shown by the dotted lines in the figure.

\section{Spin-parity-helicity analysis}

Following the model proposed by the TASSO Collaboration [2], we consider $\rho \rho$ production in different spin-parity and helicity states $\left(J^{P}, J_{z}\right)$, together with an isotropic production of four pions, denoted as " $4 \pi$ ". All states are assumed to be produced incoherently, and therefore no interference effects between the final states are taken into account. However, since states of different spin-parity and helicity are orthogonal, all interference terms vanish on integrating over the angular phase space. Isotropic $\rho \pi \pi$ production, included in previous analy- 

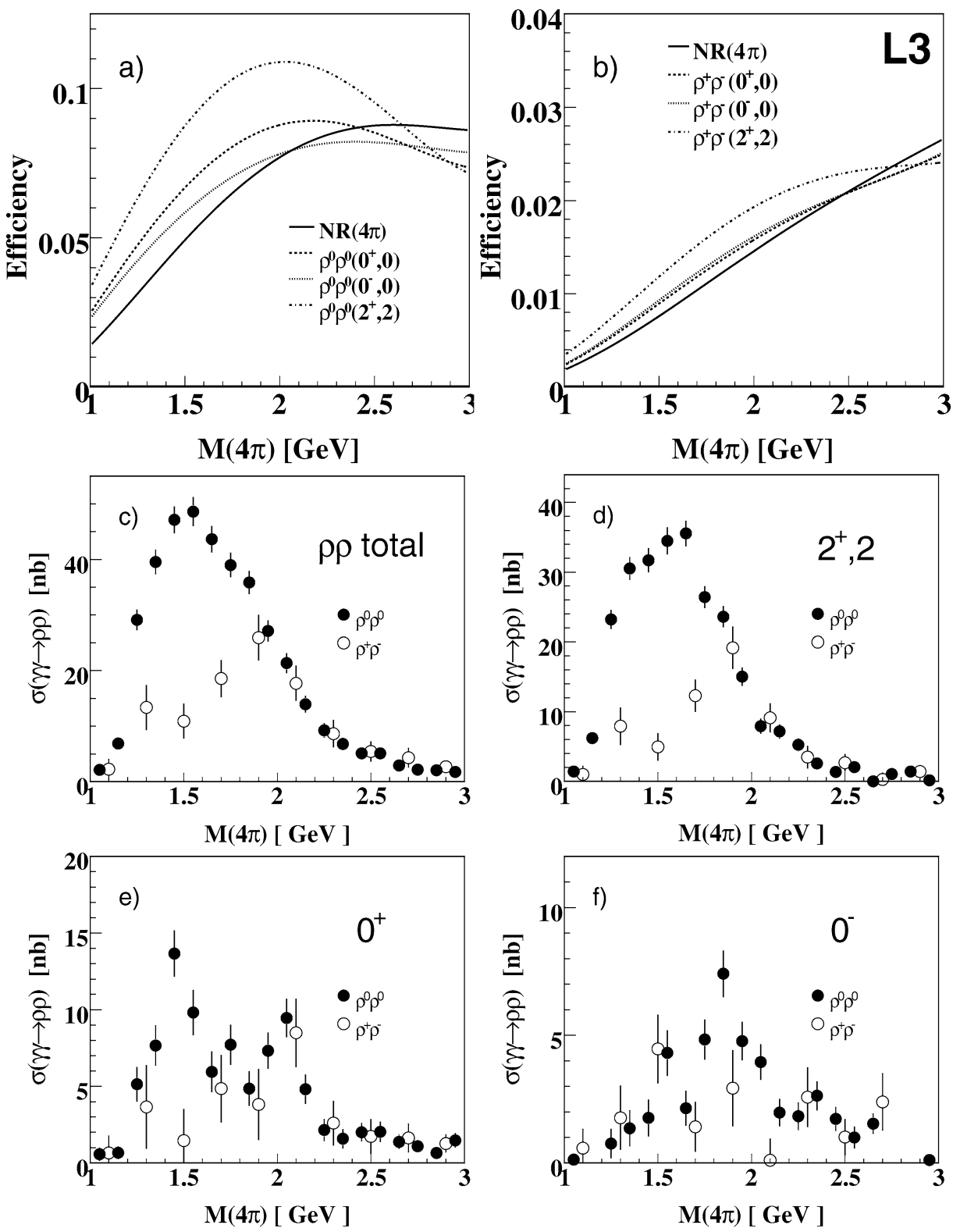

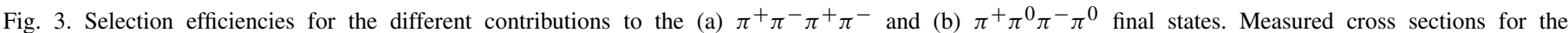

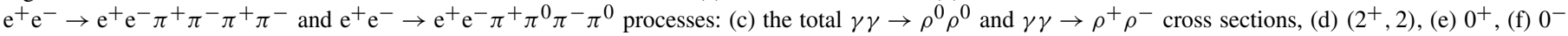
contributions. The error bars show the statistical uncertainties.

ses $[3,5]$, corresponds to an unphysical state since $C$-parity requires the angular momentum between the two pions to be odd. We have verified that this state is not essential to reproduce the data. The $\rho \pi \pi$ events, if neglected, are absorbed by the $4 \pi$ background.

The analysis is performed in $W_{\gamma \gamma}$ intervals of $100 \mathrm{MeV}$ for $\gamma \gamma \rightarrow \pi^{+} \pi^{-} \pi^{+} \pi^{-}$and $200 \mathrm{MeV}$ for $\gamma \gamma \rightarrow \pi^{+} \pi^{0} \pi^{-} \pi^{0}$. As pions are bosons, the amplitudes which describe the process must be symmetric under interchange of two pions with the same charge and are:

$g_{J^{P} J_{z}}=B_{\rho}\left(m_{\rho_{1}}\right) B_{\rho}\left(m_{\rho_{2}}\right) \Psi_{J^{P} J_{z} L S}\left(\rho_{1}, \rho_{2}\right)+$ permutations,

and $g_{4 \pi}=1$,

where $m_{\rho}$ indicates the mass of the two-pion system and $B_{\rho}\left(m_{\rho}\right)$ is the relativistic Breit-Wigner amplitude for the $\rho$ meson [21]. The angular term $\Psi_{J^{P} J_{z} L S}\left(\rho_{1}, \rho_{2}\right)$ describes the rotational properties of the $\rho \rho$ state with spin-parity $J^{P}$ and helicity $J_{z}$. It is constructed by combining the spins of the two $\rho$ mesons, $\vec{S}=\vec{s}_{1}+\vec{s}_{2}$, with $z$ projection $M_{s}=m_{1}+m_{2}$ and then adding this to the $\rho \rho$ orbital angular momentum, $\vec{L}$, with $z$ projection $M$, to obtain the state with total angular momentum $\vec{J}$ and $z$ projection $J_{z}=M_{s}+M$ :

$\Psi_{J^{P} J_{z} L S}=\sum_{M, m_{1}} C_{L M S M_{s}}^{J^{P} J_{z}} C_{s_{1} m_{1} s_{2} m_{2}}^{S M_{S}} Y_{L M}\left(\xi_{1}\right) Y_{s_{1} m_{1}}\left(\xi_{2}\right) Y_{s_{2} m_{2}}\left(\xi_{3}\right)$, where $C_{l_{1} m_{1} l_{2} m_{2}}^{J M}$ are the Clebsch-Gordan coefficients, $Y_{l m}\left(\xi_{i}\right)$ are the spherical harmonics and $\xi_{1}=\left(\vartheta_{\rho}, \varphi_{\rho}\right), \xi_{2}=\left(\vartheta_{\pi_{1}^{+}}, \varphi_{\pi_{1}^{+}}\right)$ and $\xi_{3}=\left(\vartheta_{\pi_{3}^{+}}, \varphi_{\pi_{3}^{+}}\right)$, with $\vartheta_{\rho}$ and $\varphi_{\rho}$ being the polar and azimuthal angles of a $\rho$ meson in the two-photon helicity sys- 

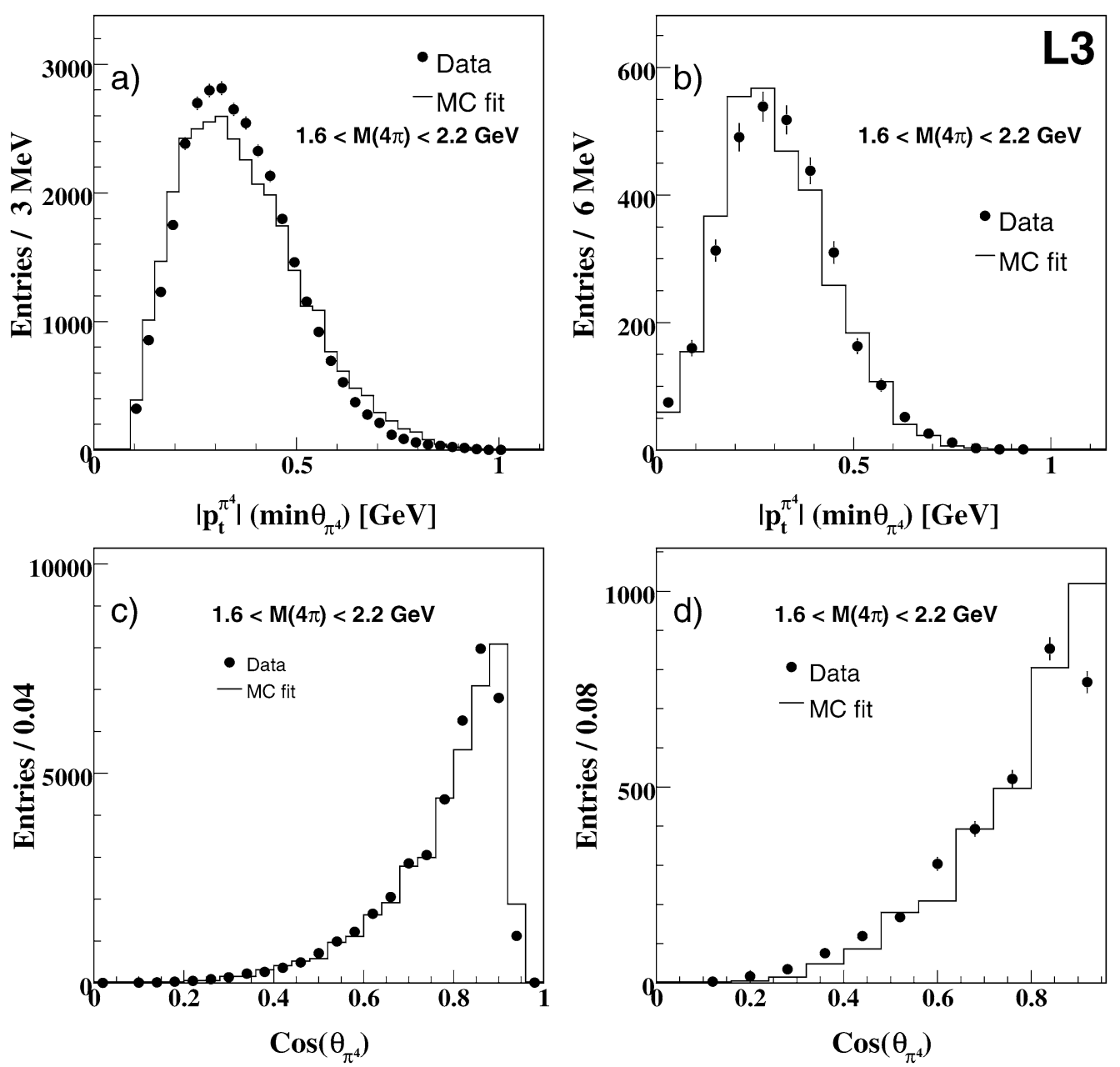

Fig. 4. Comparison of the Monte Carlo simulation normalised to the fit results to the data: (a) and (b) transverse momentum of the charged or neutral pion closest to the beam line, for $\gamma \gamma \rightarrow \pi^{+} \pi^{-} \pi^{+} \pi^{-}$and $\gamma \gamma \rightarrow \pi^{+} \pi^{0} \pi^{-} \pi^{0}$ respectively; (c) and (d) cosine of the polar angle of the charged or neutral pion closest to the beam line, for $\gamma \gamma \rightarrow \pi^{+} \pi^{-} \pi^{+} \pi^{-}$and $\gamma \gamma \rightarrow \pi^{+} \pi^{0} \pi^{-} \pi^{0}$ respectively. The statistical uncertainty on the Monte Carlo distributions-not shown-is comparable to that of the data.

tem. The $z$ axis is chosen parallel to the beam direction, which to a good approximation is parallel to the $\gamma \gamma$ helicity axis. The angles $\vartheta_{\pi_{1}^{+}}$and $\varphi_{\pi_{1}^{+}}$are the polar and azimuthal angles of the positive pions in the centre-of-mass of the first $\rho^{0}$ meson, with the $z$ axis parallel to the beam axis, the angles $\vartheta_{\pi_{3}^{+}}$ and $\varphi_{\pi_{3}^{+}}$correspond to the second $\rho^{0}$ meson; for a $\rho^{-}$meson, $\xi_{3}=\left(\vartheta_{\pi_{3}^{-}}, \varphi_{\pi_{3}^{-}}\right)$. The indices from 1 to 4 refer to the four pions using the convention: $\pi_{1}^{+} \pi_{2}^{-} \pi_{3}^{+} \pi_{4}^{-}$or $\pi_{1}^{+} \pi_{2}^{0} \pi_{3}^{-} \pi_{4}^{0}$. Since the analysis is performed close to threshold, the orbital angular momenta are restricted to $L=0,1$. The allowed spin-parityhelicity final states of the $\rho \rho$ system in quasi-real two-photon reactions are then: $\left(J^{P}, J_{z}\right)=0^{+}, 0^{-},\left(2^{+}, 0\right),\left(2^{+}, \pm 2\right)$ and $\left(2^{-}, 0\right)$, with the total spin of the $\rho \rho$ meson system $S=1$ or $S=2$. States with helicity one are forbidden by helicity conservation and spin-one states by the Landau-Yang theorem [22].

A maximum-likelihood fit to the data is used in each $W_{\gamma \gamma}$ bin to determine the contributions of the four amplitudes: $4 \pi$, $0^{+}, 0^{-}$and $\left(2^{+}, 2\right)$. The remaining spin-parity states are not considered as they have a negligible contribution if included in the fit.

\section{Cross section}

The cross section for the process $k$, with fraction $\lambda_{k}$ determined from the fit, averaged over the $W_{\gamma \gamma}$ bin with $N$ events, is

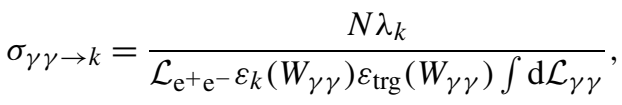

where $\int \mathrm{d} \mathcal{L}_{\gamma \gamma}$ is the two-photon luminosity function integrated over the $W_{\gamma \gamma}$ bin, $\varepsilon_{k}$ is the selection efficiency and $\varepsilon_{\text {trg }}$ is the trigger efficiency. The selection efficiencies depend on $W_{\gamma \gamma}$ as well as on the particular wave. They are computed by Monte Carlo simulation, re-weighting the events with the amplitudes $\left|g_{k}\right|^{2}$. The efficiencies for the $4 \pi$ process are listed in Tables 1 and 2. Similar efficiencies are found for the other processes. The trigger efficiency is studied by comparing the response of the two charged-track triggers. The higher-level trigger efficiencies are determined using prescaled events. The total trigger efficiency is given in Tables 1 and 2. The overall efficiencies for the $\pi^{+} \pi^{-} \pi^{+} \pi^{-}$and $\pi^{+} \pi^{0} \pi^{-} \pi^{0}$ final states are shown in Figs. 3(a) and (b). 

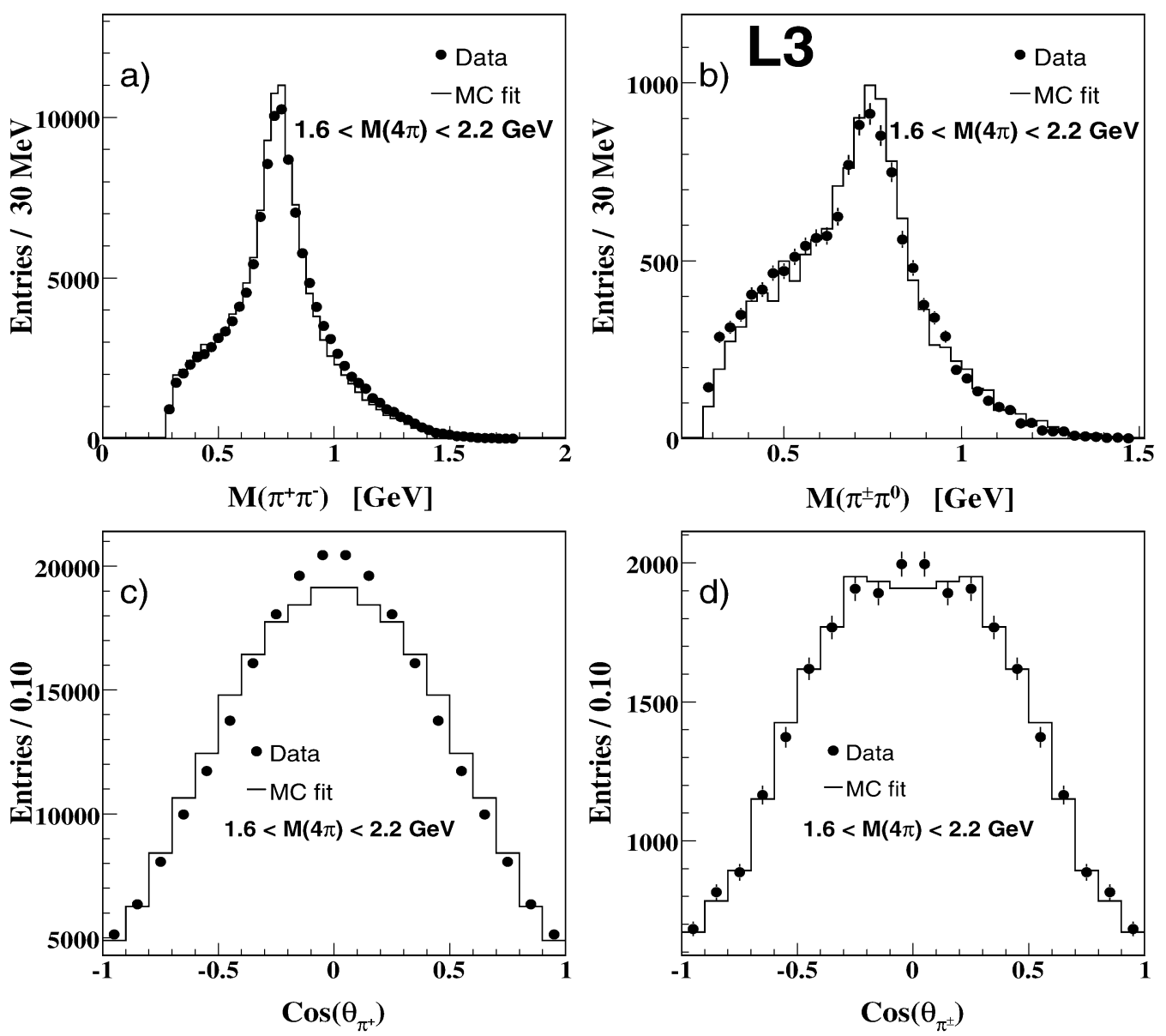

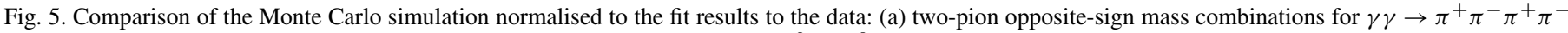

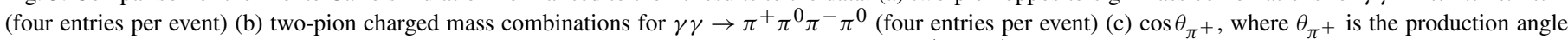

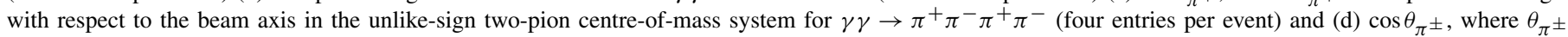
corresponds to the $\theta_{\pi}+$ angle for the $\pi^{+} \pi^{0} \pi^{-} \pi^{0}$ system (four entries per event). The error bars show the statistical uncertainties.

The cross sections derived from the fit are presented in Tables 1 and 2. Figs. 3(c)-(f) compare the total cross sections and the contributions of the individual waves to the $\gamma \gamma \rightarrow \rho^{0} \rho^{0}$ and $\gamma \gamma \rightarrow \rho^{+} \rho^{-}$processes as a function of the four-pion mass. The $4 \pi$ background, which in this analysis represents all states which do not correspond to the $\rho \rho$ production hypothesis, is similar in both channels. It grows from threshold to a value of $20-30 \mathrm{nb}$ around $2 \mathrm{GeV}$ and decreases toward $3 \mathrm{GeV}$. In the $\pi^{+} \pi^{-} \pi^{+} \pi^{-}$channel, the $\rho^{0} \rho^{0}$ production has a high cross section, with a maximum of about $50 \mathrm{nb}$ at $1.6 \mathrm{GeV}$. It is dominated by the $\left(2^{+}, 2\right)$ state, which has a cross section peak-value of about $35 \mathrm{nb}$. The $\pi^{+} \pi^{0} \pi^{-} \pi^{0}$ channel, exhibits also a significant $\left(2^{+}, 2\right)$ contribution, but only for $1.6 \leqslant W_{\gamma \gamma} \leqslant 2.2 \mathrm{GeV}$. Above $1.9 \mathrm{GeV}$ the $\gamma \gamma \rightarrow \rho^{0} \rho^{0}$ and $\gamma \gamma \rightarrow \rho^{+} \rho^{-}$cross sections are equal within the experimental uncertainties and fall rapidily with increasing $W_{\gamma \gamma}$. In the $0^{+}$wave, a clear peak is observed in the $\rho^{0} \rho^{0}$ channel at $W_{\gamma \gamma} \simeq 1.4 \mathrm{GeV}$, perhaps indicative of an $s$-channel resonance effect, it is absent in the $\rho^{+} \rho^{-}$channel. The $\gamma \gamma \rightarrow \rho^{+} \rho^{-}$cross section peaks near $2 \mathrm{GeV}$ in both $0^{+}$and $\left(2^{+}, 2\right)$ waves, while a similar peak is seen for the $\rho^{0} \rho^{0}$ in the $0^{+}$wave only. The same qualitative features were observed by the ARGUS Collaboration $[3,4]$, which however found a higher peak cross section of $\simeq 50 \mathrm{nb}$ for the $\left(2^{+}, 2\right)$ wave in $\rho^{0} \rho^{0}$. Taking into account the larger experimental uncertainties on the ARGUS data, a peak is also seen in the $0^{+}$wave at $W_{\gamma \gamma} \simeq 1.4 \mathrm{GeV}$. However, at higher mass values, $W_{\gamma \gamma}>2 \mathrm{GeV}$, only the much higher statistics of the present experiment are able to provide cross section measurements, so no comparison is possible.

To evaluate the quality of the fit and of the detector modeling we compare several distributions of the data with a Monte Carlo simulation normalized to the fit results. Fig. 4 shows the distributions of $p_{\mathrm{t}}$ and of the cosine of the polar angle of the charged or neutral pion closest to the beam line. The two-pion mass combinations, $\pi^{+} \pi^{-}$and $\pi^{ \pm} \pi^{0}$, and the production angles of the pions in the two-pion centre-of-mass system with respect to the beam direction (Adair angle) are plotted in Fig. 5. Four entries per event are considered and the data are plotted before acceptance corrections. The agreement with the Monte Carlo simulation is adequate, considering the simplicity of the model and the high statistics of the data sample. The angular distributions in Figs. 5(c) and (d) are of the general form $\sin ^{2} \theta$, indicating a dominantly transverse polarisation for the produced $\rho$. 

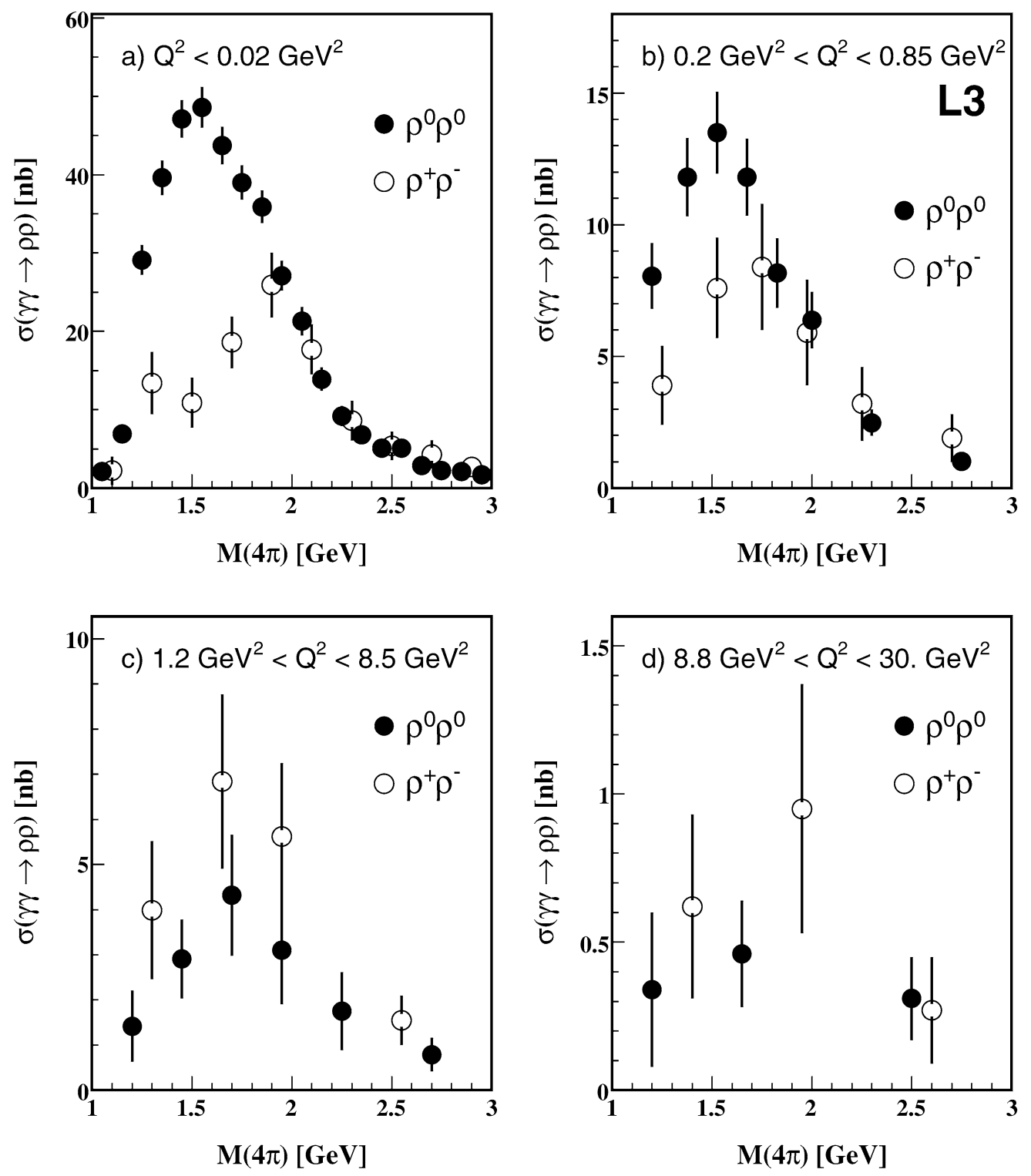

Fig. 6. The $\gamma \gamma \rightarrow \rho^{0} \rho^{0}$ and $\gamma \gamma \rightarrow \rho^{+} \rho^{-}$cross sections as a function of the four-pion mass (a) as obtained in the present analysis at $Q^{2} \leqslant 0.02 \mathrm{GeV}^{2}$ compared to previous $\mathrm{L} 3$ results obtained at (b) $0.20 \mathrm{GeV}^{2} \leqslant Q^{2} \leqslant 0.85 \mathrm{GeV}^{2}[13,14]$, (c) $1.2 \mathrm{GeV}^{2} \leqslant Q^{2} \leqslant 8.5 \mathrm{GeV}^{2}[11,12]$ and (d) $8.8 \mathrm{GeV}^{2} \leqslant Q^{2} \leqslant 30 \mathrm{GeV}^{2}[11,12]$. The error bars show the statistical uncertainties.

\section{Background estimation and systematics}

The fraction of non-exclusive background in the $\rho \rho$ sample is derived by performing a spin-parity-helicity analysis of the background data sample, defined as the region $0.2 \mathrm{GeV}^{2}<$ $\left|\Sigma \vec{p}_{\mathrm{t}}\right|^{2}<0.8 \mathrm{GeV}^{2}$. We find that less than $30 \%$ of these events are classified as $\rho \rho$. The background contribution in the $\rho \rho$ sample is then of the order of $1 \%$.

Systematic uncertainties on the $\rho \rho$ cross sections are due to selection criteria, fitting procedures and trigger efficiencies. Uncertainties from the selection procedure are estimated by varying the cuts on the quality of the event and on the track definition. They vary between $3 \%$ and $10 \%$ for the $\rho^{0} \rho^{0}$ channel and between $10 \%$ and $24 \%$ for the $\rho^{+} \rho^{-}$channel, depending on $W_{\gamma \gamma}$. Uncertainties on the model and the fitting procedure are estimated by neglecting in turn the $0^{+}$ and $0^{-}$waves and including the $\left(2^{+}, 0\right)$ and $\left(2^{-}, 0\right)$ waves in the fit. Small effects from the inclusion of additional spinparity states are also considered. In the high mass region, $W_{\gamma \gamma}>2 \mathrm{GeV}$, the contribution of other channels and higherangular momentum states may become important. It was found that such effects could be modelled by including a contribution from the isotropic $\rho \pi \pi$ production. In total, these uncertainties for the $\rho^{0} \rho^{0}$ and $\rho^{+} \rho^{-}$channels amount to a maximum of $10 \%$ and $19 \%$, respectively, for $W_{\gamma \gamma}<2 \mathrm{GeV}$ and to a maximum of $60 \%$ in the region $2 \mathrm{GeV}<W_{\gamma \gamma}<$ $3 \mathrm{GeV}$. Uncertainties on the determination of the trigger efficiencies are of a statistical nature and affect mainly the $\pi^{+} \pi^{0} \pi^{-} \pi^{0}$ channel, where they vary between $2 \%$ and $6 \%$. They are below $1 \%$ for the $\pi^{+} \pi^{-} \pi^{+} \pi^{-}$channel. Uncertainties on the background level are below $1 \%$ for both channels. 


\section{Discussion}

A spin-parity-helicity analysis of four-pion final states produced in quasi-real two-photon collisions at LEP benefits from data statistics an order of magnitude higher than previous analyses. Several characteristics of the $\gamma \gamma \rightarrow \rho^{0} \rho^{0}$ and $\gamma \gamma \rightarrow$ $\rho^{+} \rho^{-}$processes, which were previously observed [3,5], are confirmed:

- In both channels, the $\left(2^{+}, 2\right)$ wave is dominant. Small but significant $0^{+}$and $0^{-}$waves are also observed.

- The $\gamma \gamma \rightarrow \rho^{0} \rho^{0}$ process has a high cross section extending from threshold to about $2 \mathrm{GeV}$, while the cross section of the $\gamma \gamma \rightarrow \rho^{+} \rho^{-}$process is low in this range. In Fig. 6 the mass spectra of the present results are compared to those we obtained at higher $Q^{2}$ [11-14]. The ratio

$R=\sum \Delta \sigma_{\mathrm{ee}}\left(\rho^{+} \rho^{-}\right) / \sum \Delta \sigma_{\mathrm{ee}}\left(\rho^{0} \rho^{0}\right)$,

where $\Delta \sigma_{\mathrm{ee}}=\Delta \mathcal{L}_{\gamma \gamma} \sigma_{\text {tot }}(\gamma \gamma \rightarrow \rho \rho)$ and the sum is for the region $1.1 \mathrm{GeV} \leqslant W_{\gamma \gamma} \leqslant 2.1 \mathrm{GeV}$, is found to be

$R=0.42 \pm 0.05 \pm 0.09$ for $Q^{2} \leqslant 0.02 \mathrm{GeV}^{2}$.

The first uncertainty is statistical and the second systematic, calculated assuming the systematic uncertainties for the two processes to be fully uncorrelated. This ratio increases with the photon virtuality. At higher $Q^{2}$ we previously obtained:

$R=0.62 \pm 0.10 \pm 0.09$ for

$$
0.2 \mathrm{GeV}^{2} \leqslant Q^{2} \leqslant 0.85 \mathrm{GeV}^{2}[14],
$$

$R=1.81 \pm 0.47 \pm 0.22$ for

$$
1.2 \mathrm{GeV}^{2} \leqslant Q^{2} \leqslant 8.5 \mathrm{GeV}^{2}[14] \text {, }
$$

$R=2.2 \pm 1.1 \pm 0.6$ for

$$
8.8 \mathrm{GeV}^{2} \leqslant Q^{2} \leqslant 30 \mathrm{GeV}^{2}[12] .
$$

These measurements are consistent with the presence of an $s$ channel enhancement at low $\rho^{0} \rho^{0}$ mass values which decreases rapidly with $Q^{2}$. If interpreted as an effect of $s$-channel resonances, the observed ratio between $\gamma \gamma \rightarrow \rho^{+} \rho^{-}$and $\gamma \gamma \rightarrow$ $\rho^{0} \rho^{0}$ production implies the possible existence of an isospin-2 state $[8,9]$. Such an interpretation of our data was recently presented in Ref. [23].

- At higher masses, $W_{\gamma \gamma}>2 \mathrm{GeV}$, the $\gamma \gamma \rightarrow \rho^{+} \rho^{-}$and $\gamma \gamma \rightarrow \rho^{0} \rho^{0}$ cross sections are equal, within the experimental uncertainties. In both cases, the cross section decreases rapidly for $W_{\gamma \gamma} \sim 3 \mathrm{GeV}$.

The $Q^{2}$-dependence of the two-photon cross section is presented in Fig. 7 for the full mass-region $1.1 \mathrm{GeV} \leqslant W_{\gamma \gamma} \leqslant$ 3. GeV. The $\rho^{0} \rho^{0}$ cross section exceeds the $\rho^{+} \rho^{-}$one at low $Q^{2}$ while a cross-over is observed in the vicinity of $Q^{2} \simeq$ $1 \mathrm{GeV}^{2}$. A generalised vector dominance fit, GVDM [24], which reproduces well all the mid-virtuality and high-virtuality data [14] for the $\gamma \gamma \rightarrow \rho^{0} \rho^{0}$ cross section, lies below the cross section value obtained at $\left\langle Q^{2}\right\rangle=0.001 \mathrm{GeV}^{2}$. A $\rho$-pole fit, also presented in Fig. 7, better describes the low- $Q^{2}$ region.
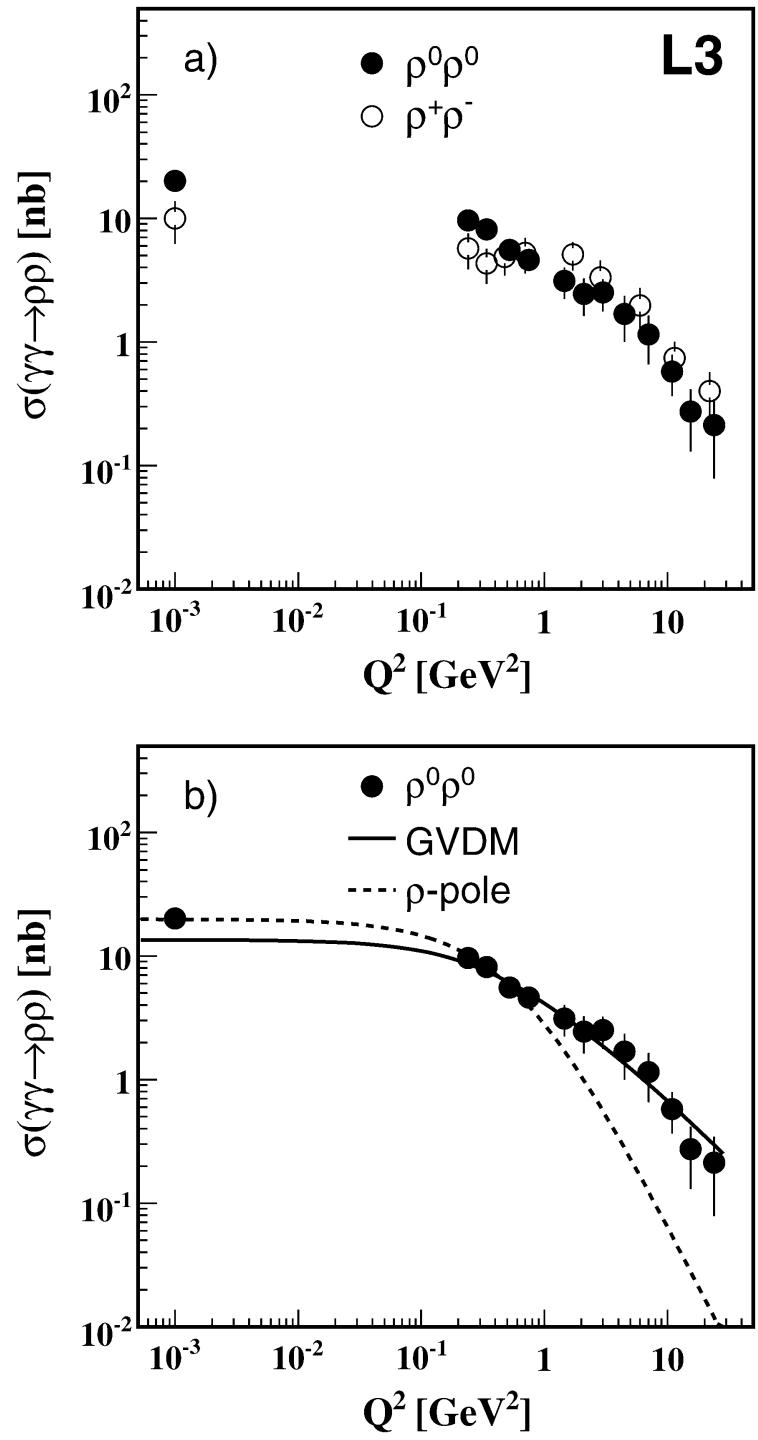

Fig. 7. (a) The $\gamma \gamma \rightarrow \rho^{0} \rho^{0}$ and $\gamma \gamma \rightarrow \rho^{+} \rho^{-}$cross sections as a function of $Q^{2}$. (b) Comparison of the $\gamma \gamma \rightarrow \rho^{0} \rho^{0}$ cross section as a function of $Q^{2}$ to a GVDM and a simple $\rho$-pole form-factor dependence, both fitted to previous L3 data at higher $Q^{2}$ [14]. The error bars show the statistical uncertainties.

\section{References}

[1] TASSO Collaboration, R. Brandelik, et al., Phys. Lett. B 97 (1980) 448; MARK II Collaboration, D.L. Burke, et al., Phys. Lett. B 103 (1981) 153; CELLO Collaboration, H.-J. Behrend, et al., Z. Phys. C 21 (1984) 205; PLUTO Collaboration, Ch. Berger, et al., Z. Phys. C 38 (1988) 521; TPC/Two-Gamma Collaboration, H. Aihara, et al., Phys. Rev. D 37 (1988) 28.

[2] TASSO Collaboration, M. Althoff, et al., Z. Phys. C 16 (1982) 13.

[3] ARGUS Collaboration, H. Albrecht, et al., Z. Phys. C 50 (1991) 1.

[4] ARGUS Collaboration, H. Albrecht, et al., Phys. Lett. B 217 (1989) 205.

[5] ARGUS Collaboration, H. Albrecht, et al., Phys. Lett. B 267 (1991) 535.

[6] J.L. Rosner, Phys. Rev. D 70 (2004) 034028, and references therein.

[7] G. Alexander, U. Maor, P.G. Williams, Phys. Rev. D 26 (1982) 1198; G. Alexander, A. Levy, U. Maor, Z. Phys. C 30 (1986) 65.

[8] B.A. Li, K.F. Liu, Phys. Lett. B 118 (1982) 435; B.A. Li, K.F. Liu, Phys. Rev. Lett. 51 (1983) 1510; B.A. Li, K.F. Liu, Phys. Rev. D 30 (1984) 613; B.A. Li, K.F. Liu, Phys. Rev. Lett. 58 (1987) 2288.

[9] N.N. Achasov, et al., Phys. Lett. B 108 (1982) 134; 
N.N. Achasov, et al., Z. Phys. C 16 (1982) 55;

N.N. Achasov, et al., Z. Phys. C 27 (1985) 99;

N.N. Achasov, et al., Phys. Lett. B 203 (1988) 309.

[10] L3 Collaboration, B. Adeva, et al., Nucl. Instrum. Methods A 289 (1990)

35 ;

L3 Collaboration, O. Adriani, et al., Phys. Rep. 236 (1993) 1;

M. Acciarri, et al., Nucl. Instrum. Methods A 351 (1994) 30;

M. Chemarin, et al., Nucl. Instrum. Methods A 349 (1994) 345;

I.C. Brock, et al., Nucl. Instrum. Methods A 381 (1996) 236;

A. Adam, et al., Nucl. Instrum. Methods A 383 (1996) 342.

[11] L3 Collaboration, P. Achard, et al., Phys. Lett. B 568 (2003) 11.

[12] L3 Collaboration, P. Achard, et al., Phys. Lett. B 597 (2004) 26.

[13] L3 Collaboration, P. Achard, et al., Phys. Lett. B 604 (2004) 48.

[14] L3 Collaboration, P. Achard, et al., Phys. Lett. B 615 (2005) 19.
[15] F.L. Linde, Charm production in two-photon collisions, Ph. D. Thesis, Rijksuniversiteit Leiden, 1988.

[16] V.M. Budnev, et al., Phys. Rep. 15 (1974) 181.

[17] R. Brun et al., preprint CERN DD/EE/84-1 (1984), revised 1987.

[18] H. Fesefeldt, RWTH Aachen report PITHA 85/2, 1985.

[19] P. Béné, et al., Nucl. Instrum. Methods A 306 (1991) 150.

[20] D. Haas, et al., Nucl. Instrum. Methods A 420 (1999) 101.

[21] J.D. Jackson, Nuovo Cimento 34 (1964) 1644.

[22] L.D. Landau, Dokl. Akad. Nauk USSR 60 (1948) 207, English summary in Phys. Abstracts A 52 (1949) 125;

C.M. Yang, Phys. Rev. 77 (1950) 242

[23] I.V. Anikin, B. Pire, O.V. Teryaev, Phys. Lett. B 626 (2005) 86.

[24] J.J. Sakurai, D. Schildknecht, Phys. Lett. B 40 (1972) 121; I.F. Ginzburg, V.G. Serbo, Phys. Lett. B 109 (1982) 231. 